\title{
Grandezas físicas multidimensionais
}

Multidimensional physical quantities

\author{
L.M. Gaio ${ }^{1}$, D.R.T. de Barros ${ }^{1}$, B.F. Rizzuti*10 \\ ${ }^{1}$ Universidade Federal de Juiz de Fora, Departamento de Física, Juiz de Fora, MG, Brasil
}

Recebido em 23 de Outubro, 2018. Revisado em 04 de Janeiro, 2019. Aceito em 05 de Janeiro, 2019.

\begin{abstract}
No presente trabalho apresentamos a continuação do recente artigo "Grandezas Físicas Unidimensionais", publicado na Revista Brasileira de Ensino de Física. A construção operacional de uma grandeza passa pela definição de seu domínio, separação do domínio em classes e, por fim, associação injetiva de um espaço de valores às classes. Essa formulação é aqui delineada para abarcar grandezas multidimensionais, incluindo vetores, vetores duais e tensores. Adotar um viés operacional, nesse caso, permite ressignificar assuntos aparentemente tautológicos da matemática, como geometria e topologia, a partir de sua conexão com o mundo físico.
\end{abstract}

Palavras-chave: Grandezas físicas, Operacionalismo, Teoria da Medida.

In the present paper we present the continuation of the recent paper "One-dimensional physical quantities", published in Revista Brasileira de Ensino de Física. The operational construction of a physical quantity passes through the definition of its domain, separation of the domain into equivalence classes and, finally, the injective association of equivalence classes into a value space. This formulation is outlined here to encompass multidimensional quantities, including vectors, dual vectors and tensors. Adopting an operational bias, in this case, allows us to re-signify seemingly tautological topics of mathematics, such as geometry and topology, from its connection to the physical world.

Keywords: Physical Quantities, Operationalism, Measurement theory.

\section{Introdução}

Este trabalho é uma continuação natural do artigo "Grandezas Físicas Unidimensionais" [1], recentemente publicado na Revista Brasileira de Ensino de Física. Lá, apresentamos com detalhes a construção do conceito de uma grandeza física. Nesse caso, em particular, discutimos sobre três delas, pilares para a mecânica clássica: distância, massa e tempo. Tal construção pode ser resumida por uma sequência de passos, que passamos a descrever. Usaremos a grandeza distância para elucidar nossa discussão inicial. Em primeiro lugar, fixada uma grandeza $G$, caracteriza-se o que chamamos de domínio da grandeza, denotado por $D_{G}$. O domínio nada mais é que o conjunto de objetos para o qual a grandeza tem sentido. Por exemplo, distância é definida para pares de pontos. Feito isso, precisamos de um procedimento experimental que caracterize grandezas do mesmo tipo. Tal comparação no caso de distâncias é feita por um compasso. Dizemos que um par de pontos define a mesma distância que um segundo par, quando for possível colocar as pontas de um compasso sobre o primeiro par e, sem alterar sua abertura, colocá-las também sobre o segundo par. Tal procedimento, que chamaremos de $\sim$, pode ser encarado como uma relação no sentido matemático, isto é, é um subconjunto do produto cartesiano $D_{G} \times D_{G}$, já que estamos comparando sempre pares de elementos. O fato

*Endereço de correspondência: brunorizzuti@ice.ufjf.br é que $\sim$ separa $D_{G}$ em classes de objetos do mesmo tipo. Por fim, associamos cada classe com um valor numérico oriundo de um conjunto que chamaremos $V_{G}$, o conjunto de valores da grandeza. Essa associação corresponde à medida como usualmente a conhecemos. A possibilidade de fazer previsões e estimativas, em um certo sentido, nos exige definir uma soma e multiplicação por números em $V_{G}$, dotando tal conjunto com uma estrutura de espaço vetorial (para o caso de distâncias, essas operações serão revistas na Seção 2). Curiosamente, a base do espaço $V_{G}$ corresponde às unidades da grandeza. Toda essa construção é ilustrada na Figura 1

Parte da motivação para o trabalho 1] foi completar a definição de um grandeza física fornecida pelo Joint Committee for Guides in Metrology [2]. Além disso, toda a construção é fortemente baseada no formalismo operacionalista [3, 4]. Partimos da premissa que não podemos usar nenhum conceito sem que seja possível defini-lo operacionalmente, isto é, a partir de um procedimento experimental. Embora a perspectiva operacionista ter sido superada em filosofia da ciência, não podemos subestimar seu potencial pedagógico. Acreditamos que adotá-la pode revelar como a física e a matemática estão conectadas, devido ao seu caráter "lúdico" destacado exatamente pela exigência de definições operacionais.

Até agora, só mencionamos grandezas unidimensionais. Contudo, já no ensino médio e nas disciplinas iniciais dos cursos de graduação em ciências exatas, nos depara- 


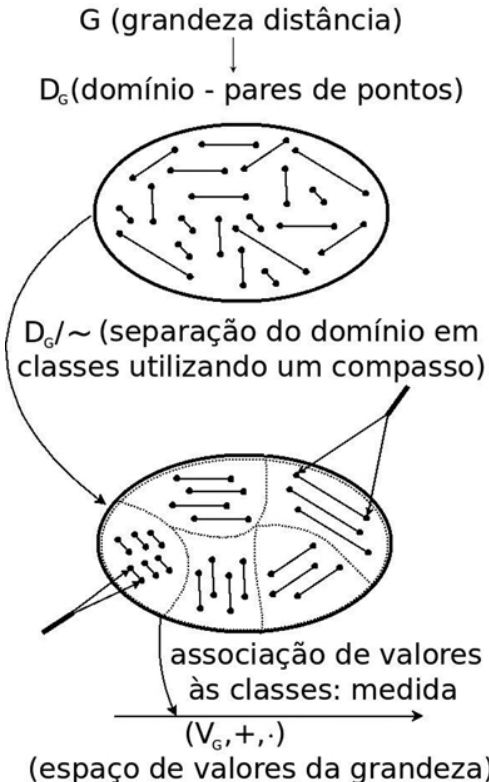

Figura 1: Representação simbólica da construção da grandeza física distância.

mos com grandezas que não são unidimensionais e elas possuem um papel central na descrição de toda a física, seja nos regimes quântico, clássico ou relativístico. São elas os vetores, vetores duais e tensores. Será que essas grandezas podem ser caracterizadas seguindo os mesmos passos delineados acima e extensamente descritos em [1]? Nosso objetivo central neste trabalho será responder a esta pergunta, caracterizando tais grandezas multidimensionais, essencialmente geométricas, seguindo uma prescrição operacional.

Adotar a corrente filosófica operacional pode ser um tanto controversa e não é, naturalmente, a única maneira de se apresentar determinado modelo. Contudo, temos aqui a chance de advogar um rompimento brusco com a geometria, puramente estruturada por uma sequência de axiomas e regras de lógica, que juntos fornecem todos os resultados que um matemático poderia encontrar. Nesse sentido, tais resultados são tautológicos, "por conterem nada mais do que já está contido nas premissas ou nos axiomas" 5]. Adotando o operacionalismo, somos capazes de reestruturar a geometria, definindo as estruturas básicas como pontos, retas e planos, sem ter que caracterizá-las como conceitos primitivos ou termos indefinidos [6], gerando assim resultados "de fatos observados, que então nunca são certos, mas somente verificáveis para um número finito de exemplos" [5]. Como veremos também, é possível, sob tal ponto de vista operacional, construir noções mais avançadas de matemática, incluindo, por exemplo, a topologia do espaço. O operacionalismo permite ainda, logo nos cursos iniciais de física, colocar o estudante de frente para um dos conceitos fundamentais da matemática e das ciências quantitativas, a saber, o conceito de linearidade. Além disso, evidencia de forma totalmente elegante a relevância dos espaços afins na física e a construção de vetores de maneira independente de coordenadas, em contraste com a comunidade matemática em geral.
Este trabalho será então dividido da seguinte maneira. Na Seção 2, apresentamos a construção básica de uma geometria primordialmente operacional, que servirá de alicerce para todo o trabalho. Na Seção 3 tratamos a primeira grandeza multidimensional, os vetores. Para ampliar e completar a estrutura da geometria do espaço discutida anteriormente, descreveremos ainda a grandeza unidimensional ângulo, seguido de algumas de suas consequências. As Seções 5 e 6 continuam com grandezas multidimensionais, a saber, vetores duais e tensores. Finalmente delineamos as conclusões na Seção 7

Ao longo de todo o trabalho, sempre que algo for definido com uma equação, utilizaremos o símbolo :=.

\section{Ingredientes básicos: espaços, pontos, retas e planos}

A primeira grandeza multidimensional que construiremos serão vetores. Eles possuem um caráter essencialmente geométrico. Assim, faremos um prelúdio na geometria. Trataremos de conceitos extremamente familiares, em particular, de espaços, pontos, retas e planos. A razão para discutirmos estas estruturas básicas é que os matemáticos as tomam como conceitos primitivos, e unindo regras de lógica e uma sequência de axiomas, constroem o que conhecemos como geometria plana [6]. Esta metodologia é ortogonal à postura operacional adotada aqui. Assim, buscaremos definições operacionais para tais conceitos 7 .

\subsection{Pontos, corpos rígidos e espaço}

Começamos com a seguinte

Definição 1 Chamaremos de pontos a interseção de riscos em cruz feitos com agulhas em corpos.

Apesar de ingênua, esta definição é adotada tácita e usualmente. De fato, quando vamos furar uma parede para prender um quadro ou mesmo serralheiros e carpinteiros a utilizam cotidianamente em suas oficinas.

Com a Definição 1 em mãos, temos um fato experimental que resumiremos em um

Axioma 1 Existem corpos na natureza nos quais a distância entre pontos marcados não muda com o tempo. Tais corpos serão chamados de referência rígidos, ou simplesmente, corpos rígidos.

A sequência de imagens na Fig. 2 ilustra este axioma.

Um contraexemplo seria a marcação com um prego de pontos $A$ e $B$ sobre a lataria de um carro em um dia de inverno. Poderíamos usar um compasso de madeira para evitar efeitos de dilatação, ou mesmo uma régua de acrílico. A medição da distância entre os pontos $A$ e $B$ em um dia quente de verão não fornece a mesma medida.

Podemos agora unir corpos de referência rígidos com uma cola que não deixe que eles se movam. Ganhamos com isso um novo corpo de referência que nos permite a marcação de mais e mais pontos, com distâncias entre 


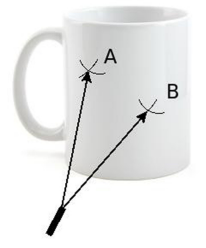

Hoje
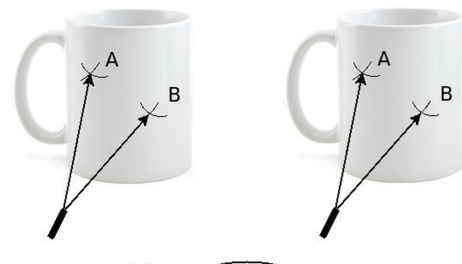

Passados 2 anos

Figura 2: Distância entre pontos em um corpo rígido não muda com o tempo.

eles cada vez maiores. Por exemplo, tijolos são corpos de referência rígida. Ao serem unidos com cimento para formar uma parede, a própria parede passa a ser um novo corpo rígido. Vamos chamar tal união de corpos rígidos de firme. Diremos resumidamente que dois corpos rígidos estão unidos de maneira firme quando a distância entre qualquer ponto do primeiro corpo até qualquer ponto do segundo não muda com o tempo. Consideremos agora o conjunto de todos os corpos rígidos.

Teorema 1 A união firme de corpos rígidos é uma relação de equivalência.

De fato, um corpo por si só já é rígido, logo tal relação é reflexiva. Sendo a distância entre pares de pontos tanto simétrica quanto transitiva, importamos tais propriedades para a união firme, caracterizando-a como uma relação de equivalência. $\left.\right|^{1}$

Denominaremos as classes definidas pela relação de equivalência de união firme de corpos rígidos como um sistema de referência rígido ou abreviadamente, sistema de referência (SR).

Consideremos o conjunto de pontos que podem ser marcados em um sistema de referência, ou simplesmente usamos nossa abstração para imaginarmos aquele estoque ilimitado de pontos que poderiam ser marcados em corpos rígidos imaginários que pertencem a determinado sistema de referência. Com isso, ganhamos a

Definição 2 O conjunto de pontos marcados em um sistema de referência (ou em corpos rígidos imaginários que pertencem a tal sistema de referência) leva o nome de espaço.

Usaremos a notação $\mathcal{E}_{S R}$ para denotar o espaço construído a partir de certo sistema de referência. Aqui já notamos um rompimento com a noção de espaço absoluto newtoniano. Com efeito, dois sistemas de referência distintos definem espaços distintos. Existem ainda dois subconjuntos de pontos em $\mathcal{E}_{S R}$ que são de interesse e serão definidos: retas e planos.

\subsection{Retas e planos}

A noção de uma reta é intimamente ligada à grandeza física "distância". Todos os detalhes podem ser vistos na

${ }^{1}$ Para o leitor não familiar ao conceito de relação e classes de equivalência, sugerimos a referência [8], que é suficientemente formal. Para uma interpretação mais intuitiva, veja [1]. subseção 2.1 de 1]: lá definimos o domínio de tal grandeza, que são pares de pontos. A relação de equivalência que divide $D_{L}$ em pares de distâncias equivalentes é feita por um compasso e detalhamos também a soma em $V_{L}$. Para dar continuidade à leitura, repetiremos a construção da soma $+: V_{L} \times V_{L} \rightarrow V_{L}$. O ponto de partida é dotar $V_{L}$ com uma relação de ordem. Inicialmente dados $A, B$ e $C$ pontos em $\mathcal{E}_{S R}$, com um compasso com abertura definida pela distância $d(A, B)$ construímos o conjunto

$$
\mathcal{B}(O, d(A, B))=\left\{X \in \mathcal{E}_{S R} \mid d(O, X)=d(A, B)\right\} .
$$

$\mathcal{B}(O, d(A, B))$ é chamado de esfera de centro $O$ e raio $d(A, B)$. Agora, dado um ponto $H \notin \mathcal{B}(O, d(A, B))$ arbitrário, colocamos uma das agulhas do compasso em $O$, e sem retirá-la do corpo rígido, fazemos um risco que conecte $O$ a $H$. Caso isso seja possível, sem que a agulha atravesse $\mathcal{B}(O, d(A, B))$, dizemos que $d(O, H)<d(A, B)$. Do contrário, $d(O, H)>d(A, B)$. A Figura 3 ilustra tal relação de ordem. Neste caso, $d(A, B)>d(O, E)$ e $d(A, B)<d(O, G)$. Agora, para obter $d(A, B)+$ $d(C, D)$, marcamos o conjunto $B\left(O^{\prime}, d(C, D)\right)$, com $O^{\prime} \in \mathcal{B}(O, d(A, B))$ e procuramos $M \in \mathcal{B}\left(O^{\prime}, d(C, D)\right)$ tal que a distância $d(O, M)$ é máxima. Assim, definimos

$$
d(A, B)+d(C, D):=d(A, M)
$$

Com isso ganhamos naturalmente a desigualdade triangular

$$
d(O, G)+d(G, M) \geqslant d(O, M)
$$

válida para pontos arbitrários do espaço. Nos interessa mesmo a igualdade, cuja interpretação geométrica é oriunda da própria definição 2. O fato mais intrigante é que passando uma linha inextensível por $O$ e $M$, mantendoa tensionada, ela passa exatamente sobre $O^{\prime}$ : veja a Figura 3. Daí diremos que $O, O^{\prime}$ e $M$ estão alinhados.

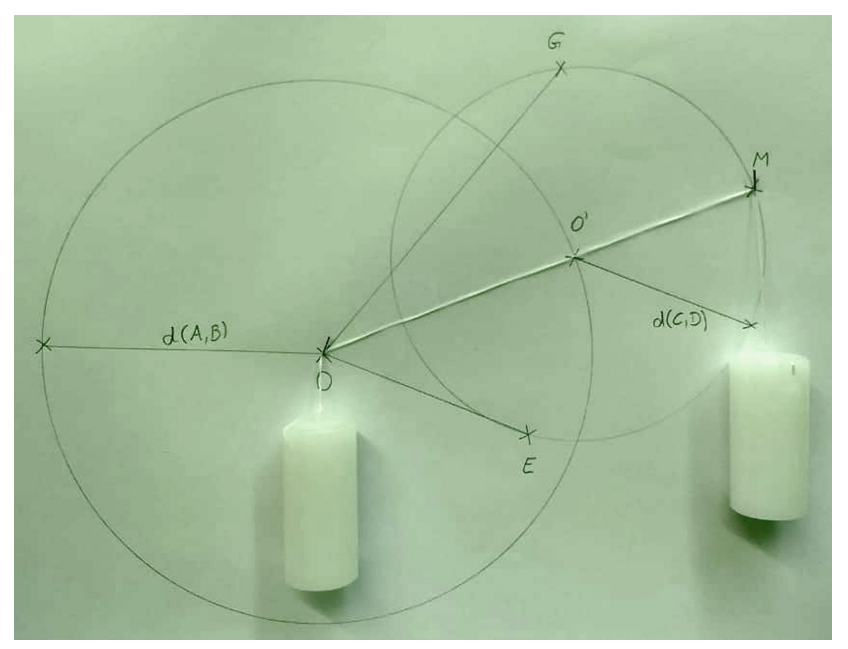

Figura 3: Ilustração da construção de (i) soma de distâncias, (ii) desigualdade triangular, (iii) segmento de reta e (iv) alinhamento de pontos. 
Além disso, diremos também que $O^{\prime}$ está entre $O$ e $M$. Finalmente estamos aptos a definir uma reta.

Definição 3 Dados $A \neq B \in \mathcal{E}_{S R}$, chamamos de reta que passa por $A$ e $B$, denotada por $r_{A B}$ o conjunto de pontos $X$, denotado por $r(X A B)$, tal que $A$ está entre $X$ e $B$, unidos com os pontos $Y$ do conjunto $r(A Y B)$, com $Y$ entre $A$ e $B$, finalmente unindo com todos os pontos $Z$ de $r(A B Z)$, tal que $B$ está entre $A$ e $Z$,

$$
r_{A B}=r(X A B) \cup r(A Y B) \cup r(A B Z) .
$$

O conjunto de pontos entre $A$ e $B$ é chamado de segmento de reta.

$\mathrm{Na}$ prática, a construção operacional de uma reta, ou melhor, de determinado segmento, é feita esticando uma linha inextensível sobre dois pontos arbitrários, o que também pode ser visto na Figura $3^{2}$

Uma vez definida o que é uma reta, precisaremos de mais uma definição, a de distância de ponto a reta.

Definição 4 Sejam $P \in \mathcal{E}_{S R}$ um ponto e $r \subset \mathcal{E}_{S R}$ uma reta. Dizemos que $d(P, r)=\inf \{d(X, P) \mid X \in r\}$ é a distância do ponto $P$ até a reta $r$.

Com esta definição em mãos, e em completa analogia com as esferas construídas anteriormente, definimos mais um subconjunto do espaço.

Definição 5 Sejam $d(A, B)$ uma distância entre dois pontos do espaço e $r \subset \mathcal{E}_{S R}$ uma reta. Definimos o conjunto $\mathcal{C}(r, d(A, B))=\left\{X \subset \mathcal{E}_{S R} \mid d(X, r)=d(A, B)\right\} e$ $o$ denominamos cilindro originado por $r$ e com raio $d(A, B)$.

A partir dessa definição temos o seguinte fato experimental expresso no

Teorema 2 Dado um ponto $P$ arbitrário em $\mathcal{C}(r, d(A, B))$, existe uma única reta $s \subset \mathcal{C}(r, d(A, B))$ e contendo $P$ tal que $d(R, s)=d(A, B), \forall R \in r$.

A interpretação geométrica deste teorema pode ser vista na Figura 4 Como consequência dele, nota-se que $d(R, s)=$ $d(A, B), \forall R \in r$ e também $d(S, r)=d(A, B), \forall S \in s$. Neste caso, chamaremos as retas $r$ e $s$ paralelas.

Finalmente, a última definição que ainda precisamos descrever operacionalmente é a de plano.

Definição 6 Sejam $r, s \subset \mathcal{E}_{S R}$ duas retas e $P \in s$ um ponto arbitrário de $s$. Definimos o conjunto $\Pi=r_{X P} \cup$ $s, \forall X \in r$, onde $r_{X P}$ é a reta que passa por $P$ e $X \in r$, como sendo o plano formado pelas retas $r$ e $s$.

A Figura 5 ilustra essa construção.

Com essas definições completamos a construção do espaço, e partimos para o estudo das grandezas físicas oriundas desta etapa inicial. Tais grandezas são essencialmente geométricas, o que justifica toda o desenvolvimento acima.

\footnotetext{
${ }^{2}$ Utilizamos um pedaço de fio dental como fio inextensível para representar o segmento de reta, preso a dois pesos plásticos.
}

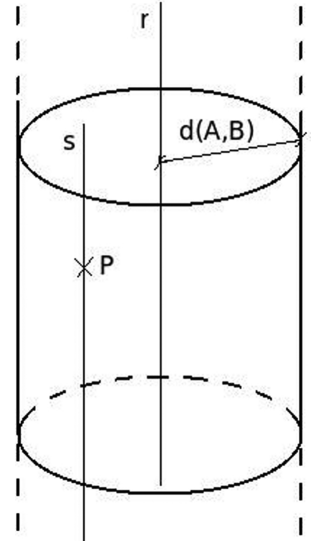

Figura 4: Representação do Teorema 2.

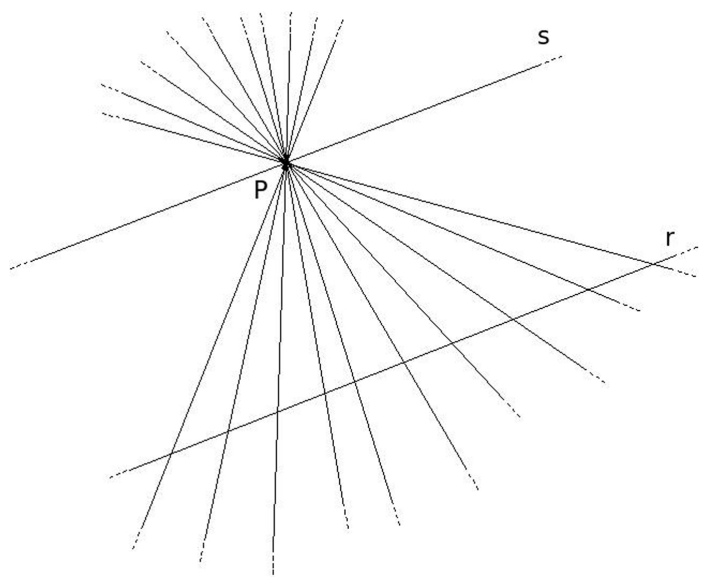

Figura 5: Definição operacional de plano.

\section{Vetores}

Passaremos agora à nossa primeira grandeza multidimensional, que chamaremos de vetores deslocamento. Assim como para distâncias, o domínio dessa nova grandeza serão pares ordenados de pontos no espaço,

$$
\mathcal{P}_{(\cdot, \cdot)}=\mathcal{E}_{S R} \times \mathcal{E}_{S R}
$$

com elementos que serão denotados por $\overrightarrow{(A, B)}$ e a seta indica que o par $\overrightarrow{(B, A)}$ é diferente de $\overrightarrow{(A, B)}$. O nome vetor deslocamento nasce de uma possível interpretação física: podemos imaginar que uma partícula se deslocou de $A$ para $B$ em linha reta. Assim, chamaremos a reta $r_{A B}$ de reta suporte de $\overrightarrow{(A, B)}$. Os pontos $A$ e $B$ serão denotados por origem e extremidade do par correspondente. Nossa próxima etapa consiste em organizar o domínio $\mathcal{P}_{(\cdot, \cdot)}$ em classes. Isto é feito por uma relação denominada transporte paralelo. Ela será denotada por

$$
\mathrm{T} \subset \mathcal{P}_{(\cdot, \cdot)} \times \mathcal{P}_{(\cdot, \cdot)}
$$

e agora passamos a descrevê-la operacionalmente. O transporte de um par $\overrightarrow{(A, B)}$ é feito com a ajuda de 
esquadros. Colocamos um esquadro sobre o segmento de reta definido por $A$ e $B$, marcamos pontos $A^{*}$ e $B^{*}$ no esquadro justapostos a $A$ e $B$ e o deslocamos com a ajuda de outro esquadro na direção desejada, conforme indica a Figura 6.

Experimentalmente, podemos ver que se trata realmente de uma relação de equivalência, já que;

i) $\overrightarrow{(A, B)} \mathrm{T} \overrightarrow{(A, B)}$, basta não deslocar o esquadro.

ii) $\overrightarrow{(A, B)} \top \overrightarrow{(C, D)} \Rightarrow \overrightarrow{(C, D)} \top \overrightarrow{(A, B)}$, pois podemos voltar o esquadro do par $\overrightarrow{(C, D)}$ até $\overrightarrow{(A, B)}$ fazendo o mesmo deslocamento inicial em sentido contrário.

iii) $\overrightarrow{(A, B)} \top \overrightarrow{(C, D)}$ e $\overrightarrow{(C, D)} \top \overrightarrow{(E, F)} \Rightarrow \overrightarrow{(A, B)} \top \overrightarrow{(E, F)}$, o que pode ser visto na Figura 7.

Cada classe de equivalência de pares de pontos ordenados que se conectam por um transporte paralelo será denotada por $\vec{a}, \vec{b}$, etc. Com essa relação em mãos, podemos definir o conjunto $\mathbb{D}=\mathcal{P}_{(\cdot, \cdot)} / T$ para "organizar"o conjunto de pares ordenados de pontos do espaço. Seguindo com a prescrição da caracterização da nossa grandeza vetores deslocamento, o primeiro passo é definir a soma entre classes,

$$
+: \mathbb{D} \times \mathbb{D} \rightarrow \mathbb{D} .
$$

Dados $\vec{a}$ e $\vec{b}$, primeiro conectamos os pontos iniciais de $\vec{a}$ e $\vec{b}$ por um transporte paralelo a um ponto $S_{1}$ arbitrário. Depois transportamos tanto $\vec{a}$ quanto $\vec{b}$ ao longo da reta

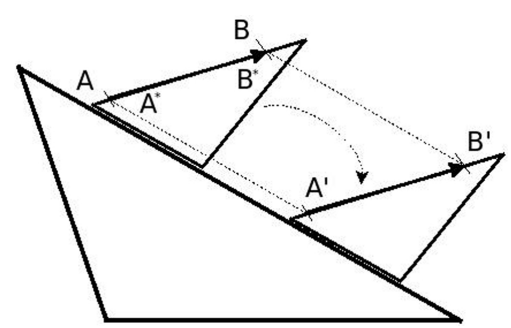

Figura 6: Transporte paralelo de um par ordenado de pontos.

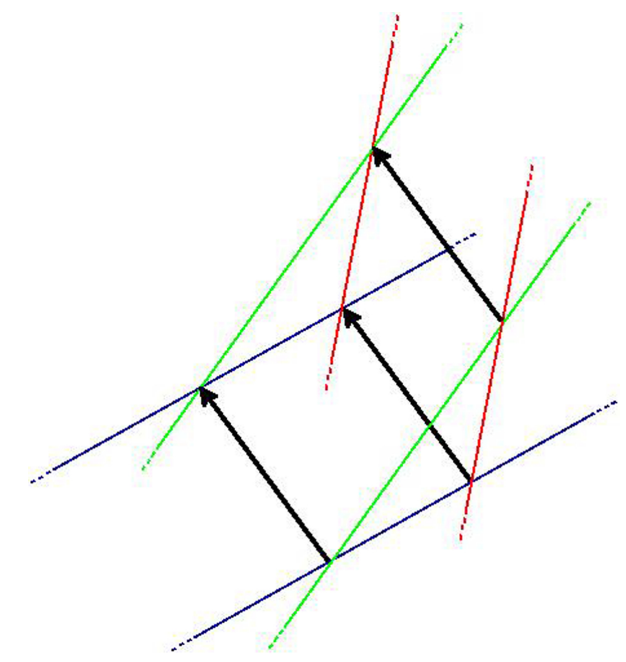

Figura 7: Transitividade do transporte paralelo. que suporta cada um deles. É um fato experimental que eles se encontram em um ponto, digamos, $S_{2}$. Chamare$\operatorname{mos} \overrightarrow{\left(S_{1}, S_{2}\right)}$ de vetor $\vec{a}+\vec{b}$. A Fig. 8 fornece o quadro geométrico desta construção. É uma tarefa rápida e direta, mostrar que a soma acima construída é uma soma de classes.

É possível também definir o produto de vetores por números reais

$$
\cdot: \mathbb{R} \times \mathbb{D} \longrightarrow \mathbb{D}
$$

Começamos com a multiplicação de um vetor $\vec{a}$ por um número natural. Ao longo da reta suporte de $\vec{a}$, marcamos com um compasso pontos consecutivos, mantendo a abertura do compasso fixa, definida pelos extremos de $\vec{a}=\overrightarrow{\left(A_{0}, A_{1}\right)}$, conforme a Figura 9 Cada ponto $A_{n+1}$ é obtido a partir de $A_{n}$. Assim, definimos

$$
n \vec{a}:=\overrightarrow{\left(A_{0}, A_{n}\right)}, \forall n \in \mathbb{N}
$$

A multiplicação por inteiros é totalmente análoga. Basta inverter o sentido de $\vec{a}$ quando o inteiro for negativo. Dado $z$ negativo em $\mathbb{Z}$, temos $z=-n$, para algum $n \in \mathbb{N}$, e então

$$
z \vec{a}=(-1) n \vec{a}=(-1) n \overrightarrow{\left(A_{0}, A_{1}\right)}:=\overrightarrow{\left(A_{n}, A_{0}\right)}
$$

Passamos agora à multiplicação por racionais $\frac{m}{n} \in \mathbb{Q}$, com $m \leq n$. Partindo de $A_{0}$, desenhamos um segmento de reta $r$ em qualquer direção diferente da reta que suporta $\vec{a}=\overrightarrow{\left(A_{0}, A_{1}\right)}$. Com um compasso com abertura arbitrária, marcamos os pontos $B_{1}, B_{2}, \ldots, B_{n}$ em $r$, de modo que $d\left(A_{0}, B_{1}\right)=d\left(B_{1}, B_{2}\right)=\ldots=d\left(B_{n-1}, B_{n}\right)$. Traçamos o segmento que conecta $B_{n}$ a $A_{1}$ e traçamos segmentos

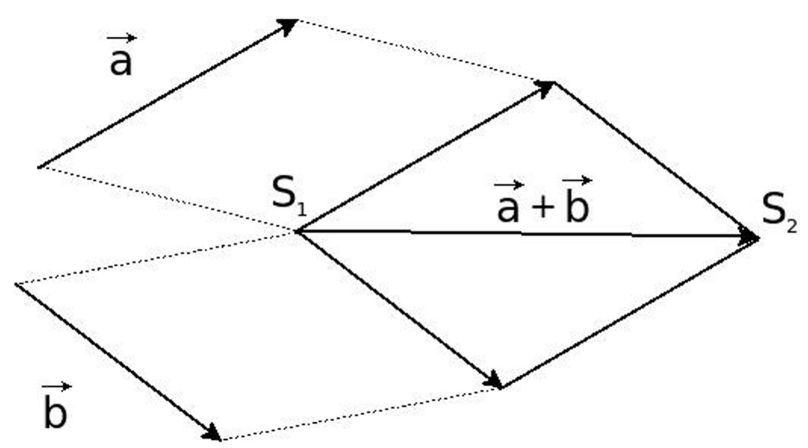

Figura 8: Construindo a soma de vetores.

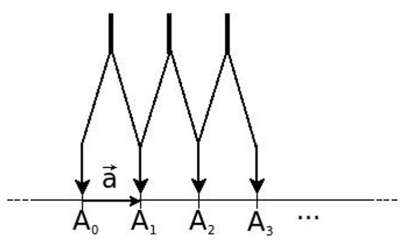

Figura 9: Construindo a multiplicação de vetores por números. 
paralelos ao segmento definido por $B_{n}$ e $A_{1}$, que vão dos pontos $B_{i}, i=1,2, \ldots, n-1$ até tocar $\vec{a}$, onde marcamos os pontos $B_{i}^{\prime}$. Assim,

$$
\frac{m}{n} \vec{a}:=\overrightarrow{\left(A_{0}, B_{m}^{\prime}\right)}
$$

A representação geométrica para tal definição da multiplicação de $\vec{a}$ por $3 / 5$ pode ser vista na Figura 10 A generalização para $m>n$ é direta. Escrevamos $m=n+m^{\prime}$, de modo que $m / n=1+m^{\prime} / n$, com $m^{\prime}<n$. Assim, basta aplicar os resultados acima discutidos.

A multiplicação por reais em $\mathbb{D}$ pode ser generalizada a partir da densidade de $\mathbb{Q}$ em $\mathbb{R}$. Contudo, observamos que temos uma limitação experimental para a construção de uma distância dada por um irracional. Com efeito, meça com uma régua a diagonal de um quadrado com lados de $1 \mathrm{~m}$. O que você encontrá? $\sqrt{2} \mathrm{~m}$ ? Ou $1,41 \mathrm{~m}$ ? Uma discussão mais detalhada sobre incertezas pode ser vista em [1].

Agora, com a soma e multiplicação por números, temos mais um fato experimental: $(\mathbb{D},+, \cdot)$ é um espaço vetorial tridimensional e ter chamado os pares ordenados de vetores anteriormente não foi coincidência. Concluímos essa Seção com um último fato experimental: o espaço físico $\mathcal{E}_{S R}$ associado a um determinado corpo de referência rígido possui as propriedades de uma estrutura muito conhecida pelos matemáticos, a saber o espaço afim. ${ }^{3}$ Ele é definido como a seguir. Sejam $E$ um conjunto cujos elementos são chamados de pontos e $V$ um espaço vetorial. Considere também uma aplicação $\overrightarrow{~: ~} E \times E \rightarrow V$, $(p, q) \mapsto \overrightarrow{p q}$. Dizemos que $E$ é um espaço afim associado ao espaço vetorial $V$ quando goza das seguintes propriedades

(i) Dados um ponto $p \in E$ e um vetor $\vec{v} \in V$ existe um único ponto $q \in E$ tal que $\overrightarrow{p q}=\vec{v}$.

(ii) Para quaisquer $p, q, r \in E$ tem-se $\overrightarrow{p q}+\overrightarrow{q r}=\overrightarrow{p r}$.

A dimensão do espaço $E$ é definida por $\operatorname{dim} E:=\operatorname{dim} V$.

O item (ii) acima é um fato experimental que mostra a bijeção entre $\mathcal{E}_{S R}$ e $\mathbb{D}$. De fato, dados dois pontos $A$ e

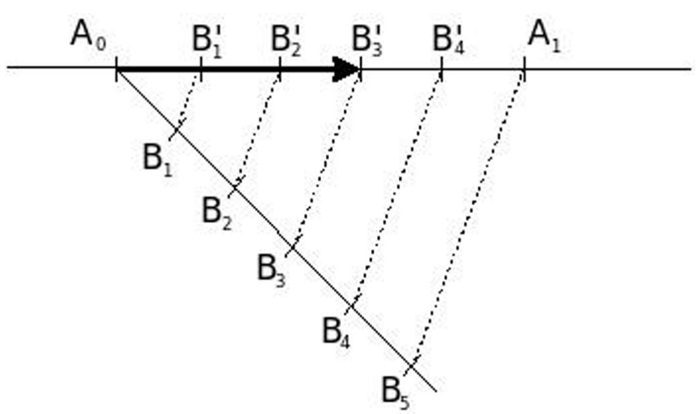

Figura 10: Representação geométrica da multiplicação de um vetor por um número racional.

\footnotetext{
${ }^{3}$ Encontramos essa mesma estrutura na teoria da relatividade. O espaço-tempo de Minkowski $\mathbb{M}$ possui a estrutura de um espaço afim quadridimensional 9 .
}

$B$ em $\mathcal{E}_{S R}$, eles definem um representante da classe de vetores deslocamento correspondente. Reciprocamente, dado um ponto $A \in \mathcal{E}_{S R}$ e um vetor $\vec{a} \in \mathbb{D}$, transportamos paralelamente a origem de $\vec{a}$ até o ponto $A$, e olhamos para a única extremidade $B$ do representante da classe $\vec{a}$, a saber, $\overrightarrow{A B}$. Na literatura matemática, é costume escrever tal resultado como

$$
B:=A+\vec{a}
$$

Esperamos que a discussão acima explicite a diferença entre o espaço afim $\mathcal{E}_{S R}$ e o espaço vetorial associado $\mathbb{D}$, apesar da bijeção entre ambos.

\section{4. Ângulos e algumas consequências}

Antes de continuarmos com grandezas multidimensionais, descreveremos mais uma grandeza de interesse que é unidimensional. Ela não foi introduzida anteriormente pois seu domínio, como veremos, é formado por pares de vetores. Faremos como antes: a descrição formal é precedida por argumentos heurísticos, culminando com procedimentos experimentais, matematicamente formalizados. Trataremos também de alguns tópicos que estão direta ou indiretamente ligados com ângulos.

\subsection{Construindo a grandeza ângulo}

Consideremos o exemplo cotidiano de abrir a porta da geladeira para retirada de uma garrafa de água. Para economizar energia, você abre a porta somente o suficiente para retirar a garrafa. Caso você tenha que retirar uma panela, a abertura da porta deve ser maior que antes. Esse exemplo soa muito natural. Mas o que queremos dizer com abertura? Mais ainda, mesmo sem conseguir sequer definir abertura, inserimos uma relação de ordem ao dizer "abertura maior do que antes". Nosso objetivo será então formalizar esta noção de abertura, construindo a grandeza que chamaremos de ângulo.

Com o exemplo inicial, fica claro que o domínio da grandeza ângulo são pares de vetores. Restringiremos nossa definição inicial ao espaço vetorial bidimensional $\left.\mathbb{D}\right|_{\Pi}$ que tem $\Pi$ como espaço afim, isto é, para quaisquer $\left.\vec{a} \in \mathbb{D}\right|_{\Pi}$ e $P \in \Pi$, tem-se $P+\vec{a} \in \Pi$. Pela definição operacional de plano, é fácil ver que dois vetores definem um plano. Assim, escreveremos tal domínio como

$$
D_{A}=\left\{(\vec{a}, \vec{b})|\vec{a}, \vec{b} \in \mathbb{D}|_{\Pi}\right\} .
$$

Agora seguiremos uma sequência de passos experimentais para caracterização dessa nova grandeza. Dados $\vec{a}=\overrightarrow{(O, A)}$ e $\vec{b}=\overrightarrow{(O, B)}$,

i) Traçamos as retas $r_{O A}$ e $r_{O B}$;

ii) Colocamos a ponta seca do compasso em $O$ e marcamos o arco que conecta a reta $r_{O A}$ até a reta $r_{O B}$. Isto pode ser feito de duas, e somente duas maneiras, como 
indicado na Figura 11] a saber, no sentido horário ou no anti-horário;

iii) Variamos a abertura do compasso e marcamos mais arcos como indicados na etapa (ii) acima, escolhendo um dos dois sentidos.

Notavelmente, os raios $R_{1}, R_{2}, \ldots, R$ dos diferentes arcos da Figura 11 são diferentes, bem como os comprimentos dos arcos. Contudo, a abertura definida pelo par de vetores continua a mesma. Mais ainda, é um fato experimental que

$$
\frac{l_{1}}{R_{1}}=\frac{l_{2}}{R_{2}}=\frac{l_{3}}{R_{3}}=\frac{l}{R} .
$$

Ora, este será então exatamente o procedimento experimental que tanto define as classes em $D_{A}$ como fixa os valores de ângulos. De fato, sendo a razão entre comprimento de arco pelo raio independente da abertura do compasso, definimos

$$
\alpha:=\Varangle(\vec{a}, \vec{b})=\frac{l}{R} .
$$

Em (15), $l$ e $R$ correspondem ao comprimento do arco e raio como definidos pelos procedimentos experimentais (i-iii) acima. O símbolo $₹$ indica que o compasso sai da reta $r_{O A}$ e vai até a reta $r_{O B}$ no sentido anti-horário. É uma consequência de 15 que

$$
\Varangle(\vec{a}, \vec{b})=\Varangle(\vec{b}, \vec{a}) .
$$

Naturalmente 15 define o valor para a grandeza e, por construção, $\alpha \in \mathbb{R}$ (voltaremos ao assunto do espaço de valores de ângulos ao fim da Seção). Além disso, dois pares $(\vec{a}, \vec{b})$ e $(\vec{c}, \vec{d})$ são ângulo-equivalentes quando

$$
(\vec{a}, \vec{b}) \sim_{A}(\vec{c}, \vec{d}) \Leftrightarrow \Varangle(\vec{a}, \vec{b})=\Varangle(\vec{c}, \vec{d}) .
$$

Antes de avançarmos com as operações de soma e multiplicação por escalar, um comentário deve ser feito. A grandeza física distância possui pares de pontos como domínio. Assim, é preciso esclarecer o significado de "comprimento de arco". Como, em princípio, só sabemos medir

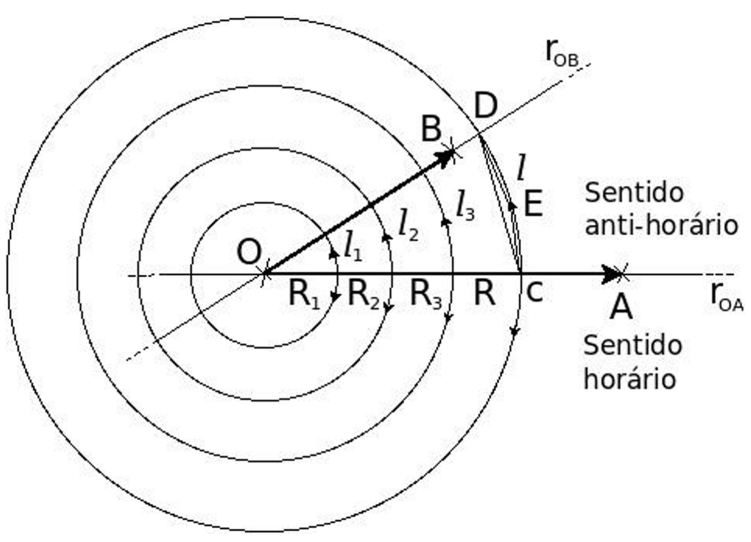

Figura 11: Construção operacional da grandeza ângulo. a distância entre dois pontos, poderíamos tentar definir o comprimento do arco descrito pelo compasso saindo de $C$ e chegando em $D$ por

$$
l:=d(C, D),
$$

onde os pontos $C$ e $D$ estão indicados na Figura 11 Mas o arco não coincide com o segmento de reta definido pelo par $C, D$. Logo, a escolha (18) não é boa. Para melhorar essa situação, colocamos um ponto a mais sobre o arco, digamos $E$, e escrevemos

$$
l:=d(C, E)+d(E, D) .
$$

Pela desigualdade triangular, $d(C, D)<d(C, E)+d(E, D)$ mas também não caímos sobre o arco com os dois segmentos definidos por $C, E$ e $E, D$. Seguimos marcando pontos $C_{i}, i=1, \ldots, N$, para algum $N \in \mathbb{N}$, sobre o arco, tão próximos quanto a menor abertura do compasso permita medir a distância $d\left(C_{i}, C_{i+1}\right)$. Escrevemos então esta soma de distâncias como

$$
S_{P}(N)=\sum_{i=1}^{N-1} d\left(C_{i}, C_{i+1}\right),
$$

com o primeiro ponto sendo $C$ e o último, $D$. Tal marcação de pontos sobre o arco é chamada de partição, justificando o índice $S_{P}$. Devido à desigualdade triangular, quanto mais pontos forem marcados,

$$
S_{P}(N) \leq S_{P}(N+1) .
$$

Tal desigualdade entre somas motiva usarmos um supremo para definir

$$
l:=\sup _{P \text { é partição }} S_{P}
$$

Dessa maneira, chegaremos o mais próximo possível do que poderemos chamar de comprimento de arco.

O fato experimental curioso é que todo o processo acima fornece o mesmo resultado, dentro da incerteza, de medida de comprimento de arco caso usássemos uma fita métrica flexível, como aquela do alfaiate. Basta, para isso, ajustar a fita sobre o arco do qual se deseja medir o comprimento, veja a Figura 12 as agulhas representam pontos de uma possível partição do arco.

Para construir a soma de ângulos, precisaremos ainda do conceito de rotação. A situação aqui é análoga à soma de distâncias. Escrever $2 \mathrm{~cm}+3 \mathrm{~cm}=5 \mathrm{~cm}$ é vazia no sentido geométrico. Com efeito, dados pontos $A, B, C$, $D$ tal que $d(A, B)=2 \mathrm{~cm}$ e $d(C, D)=3 \mathrm{~cm}$, precisamos de um procedimento para determinar qual é a localização de pontos $O$ e $M$ tal que

$$
d(A, B)+d(C, D)=d(O, M) .
$$

Com ângulos $\alpha=\Varangle(\vec{a}, \vec{b})$ e $\beta=\Varangle(\vec{c}, \vec{d})$ com $\vec{a}, \vec{b}, \vec{c}$ e $\vec{d}$ em $\left.\mathbb{D}\right|_{\Pi}$, poderíamos fazer a mesma pergunta: qual o par 


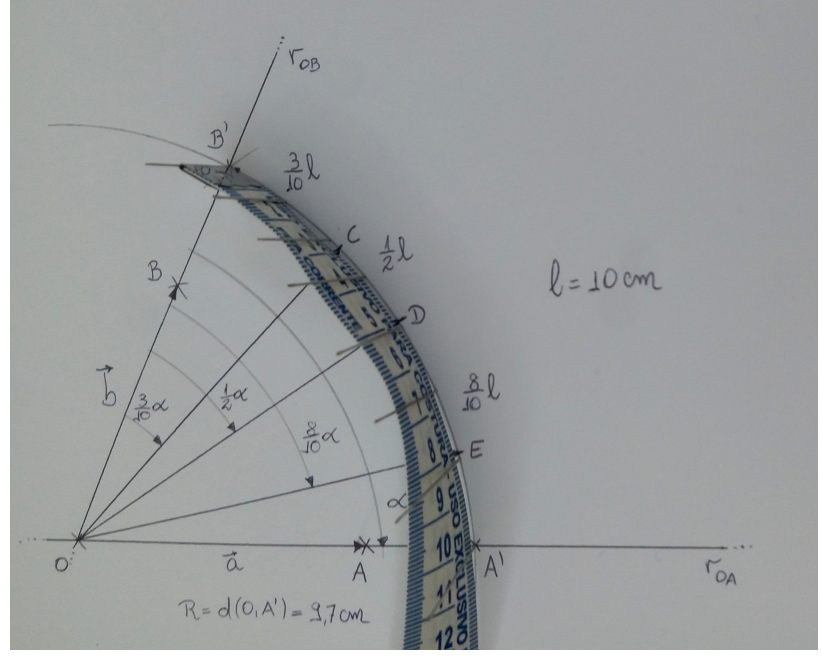

Figura 12: Representação de uma partição feita com agulhas e multiplicação de ângulos por números.

$(\vec{e}, \vec{f})$ tal que

$$
\Varangle(\vec{e}, \vec{f})=\alpha+\beta ?
$$

Para responder a essa pergunta, começamos com a

Definição 7 Dado $\Pi \subset \mathcal{E}_{S R}$, chamaremos de rotação em torno de um ponto $P \in \Pi$ o mapeamento

$$
\begin{aligned}
R_{P}: \Pi & \rightarrow \Pi \\
A & \mapsto R_{P}(A) \in \Pi
\end{aligned}
$$

tal que

i) $d(P, A)=d\left(P, R_{P}(A)\right)$;

ii) $R_{P}(P)=P$.

Reflexões por espelhos passando por $P$ preservam distâncias mas elas são excluídas da definição acima. A razão é que nosso espaço físico foi construído a partir de corpos de referência rígida. É possível girar um corpo rígido, preservando distâncias. Mas não podemos girar a mão direita para obter a esquerda; somente uma reflexão pode fazê-lo. Tecnicamente, a exigência de excluirmos reflexões significa pedir que o mapeamento $R_{P}$ seja contínuo e que possa ser deformado continuamente à identidade. Tais definições, contudo, exigem a construção de noções topológicas que não serão abordadas aqui.

Da identificação de $\mathcal{E}_{S R}$ com $\mathbb{D}$, o mapeamento $R_{P}$ induz uma rotação natural de vetores

Definição 8 Chamamos de rotação do vetor $\left.\vec{a} \in \mathbb{D}\right|_{\Pi}$ o seguinte mapeamento

$$
\begin{aligned}
\mathcal{R}:\left.\mathbb{D}\right|_{\Pi} & \left.\rightarrow \mathbb{D}\right|_{\Pi} \\
\vec{a} & \mapsto \mathcal{R}(\vec{a}):=\overline{\left(P, R_{P}(A)\right)}
\end{aligned}
$$

Notemos que não é preciso fixar o ponto pois sempre podemos transportar um elemento da classe $\vec{a}$ até o ponto $P$. A partir de agora, quando tratarmos de uma rotação, estamos fazendo referência à rotação de vetores. ${ }^{4} \mathrm{Um}$ resultado experimental associado às rotações é expresso pelo

Axioma 2 Dadas $\vec{a}$ e $\vec{b}$ classes distintas em $\left.\mathbb{D}\right|_{\Pi}$, existe uma rotação tal que $\mathcal{R}(\vec{a})$ e $\vec{b}$ são suportados por retas paralelas.

Podemos visualizar o Axioma 2 em um relógio analógico, quando o ponteiro dos segundos alcança, por exemplo, o ponteiro dos minutos.

Usando ainda a rotação de um vetor, podemos induzir a rotação de um par $\left.(\vec{a}, \vec{b}) \in \mathbb{D}\right|_{\Pi} \times\left.\mathbb{D}\right|_{\Pi}$ definida por

$$
(\vec{a}, \vec{b}) \mapsto \mathcal{R}(\vec{a}, \vec{b}):=(\mathcal{R} \vec{a}, \mathcal{R} \vec{b}) .
$$

Por construção, tal rotação preserva o valor de ângulos

$$
\alpha=\Varangle(\vec{a}, \vec{b})=\Varangle(\mathcal{R} \vec{a}, \mathcal{R} \vec{b}) .
$$

Com este arcabouço em mãos, já podemos caracterizar geometricamente a soma de ângulos. Tomemos então $\alpha=\Varangle(\vec{a}, \vec{b})$ e $\beta=\Varangle(\vec{c}, \vec{d})$. Buscamos a rotação $\mathcal{R}$ tal que $\mathcal{R}(\vec{b})$ e $\vec{c}$ sejam paralelos e com a mesma origem. Aplicamos então a rotação induzida por $\mathcal{R}$ ao par $(\vec{a}, \vec{b})$, mantendo $(\vec{c}, \vec{d})$ fixo. Definimos então

$$
\alpha+\beta:=\Varangle(\mathcal{R} \vec{a}, \vec{d}) .
$$

A Figura 13 indica essa construção.

A multiplicação de valores de ângulos por escalares é importada da própria multiplicação de números por distâncias. De fato, usamos novamente a fita métrica flexível do alfaiate, com os seguintes procedimentos. Dado certo valor de ângulo $\alpha=\Varangle(\vec{a}, \vec{b})$, desenhamos um arco arbitrário que conecte as retas que suportam os vetores $\vec{a}$ e $\vec{b}$, segundo a orientação definida ao se construir o ângulo. Medimos os comprimentos do arco $(l)$ e do raio $(R)$, de modo que $\alpha=l / R$. Dado $\lambda \in \mathbb{R}$, com $0<\lambda<1$, marcamos sobre a fita métrica flexível o valor $\lambda \cdot l$ e retornamos com

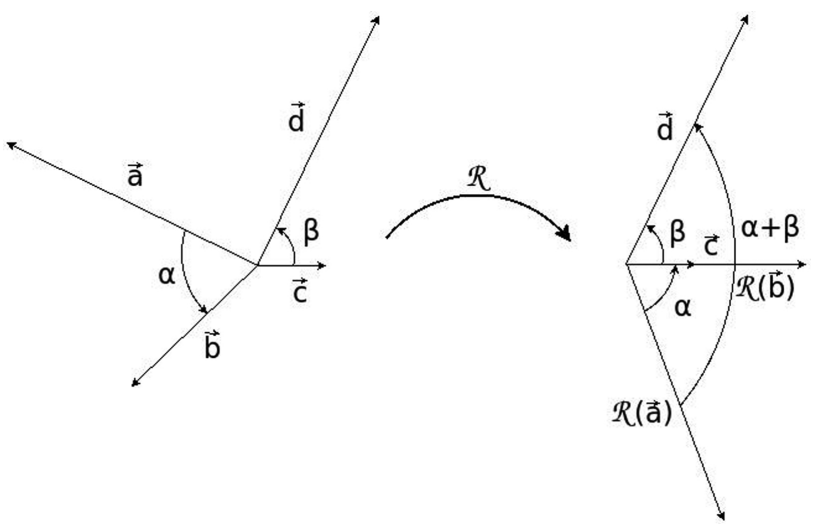

Figura 13: Quadro geométrico da soma entre ângulos.

\footnotetext{
${ }^{4}$ Rotações são descritas pela teoria de representações do grupo $S O(3)$. Os detalhes podem ser vistos em [10.
} 
a fita sobre o arco, de modo que

$$
\lambda \cdot \alpha:=\frac{\lambda \cdot l}{R} .
$$

A Figura 12 ilustra o procedimento resumido na definição (30). Nesse caso,

$$
\alpha=\Varangle(\vec{b}, \vec{a})=10 \mathrm{~cm} / 9,7 \mathrm{~cm}=1,03 .
$$

Os pontos $C, D$ e $E$ foram marcados seguindo a escala da fita métrica, de modo que

$$
\Varangle(\vec{b}, \overrightarrow{O C})=\frac{3}{10} \alpha ; \Varangle(\vec{b}, \overrightarrow{O D})=\frac{1}{2} \alpha ; \Varangle(\vec{b}, \overrightarrow{O E})=\frac{8}{10} \alpha .
$$

Devemos ter cuidado com o caso em que $\lambda<0$. Como queremos medir "aberturas", valores de ângulos negativos correspondem a caminhar com o compasso em um outro sentido. A analogia é completa ao definirmos o espaço de valores de distância. Lá podíamos multiplicar distâncias por números reais negativos e interpretar uma distância negativa como, por exemplo, uma coordenada de posição. Além disso, devido ao fato experimental que

$$
\Varangle(\vec{a}, \vec{b})+\Varangle(\vec{b}, \vec{a})=2 \pi,
$$

devemos ser cuidadosos também quando $\lambda>2 \pi$, indicando que completamos mais de uma volta. Isso pois o mesmo par de vetores pode representar mais de um valor de ângulo. Para contornar esta falta de injetividade entre classes de equivalência em $D_{A} / \sim_{A}$ definidas pela relação (17) e valores, podemos usar um índice no símbolo ₹

$$
\Varangle_{k}(\vec{a}, \vec{b})=\alpha+2 k \pi ; k \in \mathbb{Z} .
$$

Como valores de ângulo são números reais, importamos as propriedades usuais garantindo que o conjunto de valores seja um espaço vetorial. Ressaltamos ainda que para distinguir um número de outro que representa uma abertura, utiliza-se, por convenção, a palavra radiano. Isso não significa que "rad" represente unidades. A base do espaço de valores de ângulos $V_{A}$ é o conjunto unitário $\{1\} ; 1 \in \mathbb{R}$.

Com isso completamos a descrição da grandeza ângulo. As próximas subseções são dedicadas a apontamentos que estão ligados diretamente com a grandeza desenvolvida.

\subsection{Funções trigonométricas}

A ideia de ângulo surgiu quando percebemos a constância entre razão de comprimento de arco e raio, expressa por 15. De forma similar, poderíamos desenhar segmentos de retas no lugar de arcos, gerando, a partir de determinado par de vetores, triângulos com ângulos de $\pi / 2$, como indica a Figura 14 Tais ângulos são representados por um quadrado com um ponto dentro, próximo aos pontos $A_{1}, A_{2}$, etc.

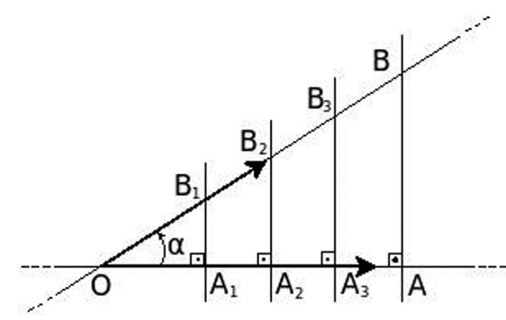

Figura 14: Construção operacional de funções trigonométricas.

O fato experimental é que, fixado o ângulo, as seguintes razões são constantes:

$$
\frac{d\left(A_{1}, B_{1}\right)}{d\left(O, B_{1}\right)}=\frac{d\left(A_{2}, B_{2}\right)}{d\left(O, B_{2}\right)}=\frac{d\left(A_{3}, B_{3}\right)}{d\left(O, B_{3}\right)}=\frac{d(A, B)}{d(O, B)} .
$$

e também

$$
\frac{d\left(O, A_{1}\right)}{d\left(O, B_{1}\right)}=\frac{d\left(O, A_{2}\right)}{d\left(O, B_{2}\right)}=\frac{d\left(O, A_{3}\right)}{d\left(O, B_{3}\right)}=\frac{d(O, A)}{d(O, B)} .
$$

Definimos, com isso, as funções de ângulo seno e cosseno, dadas pela razão entre lados de triângulos com um ângulo reto, como exposto nas expressões (35) e (36) respectivamente. Nasce, a partir daí, a teoria de funções trigonométricas com a proposta operacional.

\subsection{Dotando $\mathbb{D}$ com um produto interno}

Discutiremos agora mais uma estrutura que compõe a descrição geométrica do espaço de vetores deslocamento, o produto interno. Aparentemente não há conexão entre ângulos e produto interno, mas o Teorema 3 , a ser apresentado, justificará a inserção dessa subseção.

Como $\mathbb{D}$ foi construído a partir de pares de pontos ordenados, há uma estrutura naturalmente associada com a classe $\vec{a}$. Basta tomar qualquer representante dessa classe, digamos, $\overrightarrow{(O, A)} \in \vec{a}$ e definimos o módulo de $\vec{a}$ como

$$
\begin{aligned}
||: \mathbb{D} & \rightarrow V_{L} \\
\vec{a} & \mapsto d(O, A) .
\end{aligned}
$$

| | tem todas as propriedades para ser chamado de uma norma 11], o que pode ser prontamente verificado com um compasso. Neste caso, $\mathbb{D}$ passa a ser um espaço normado. Com o módulo em mãos, conseguimos construir um novo mapeamento sobre $\mathbb{D} \times \mathbb{D}$

$$
\begin{aligned}
\cdot: \mathbb{D} \times \mathbb{D} & \rightarrow V_{L^{2}} \\
(\vec{a}, \vec{b}) & \mapsto \vec{a} \cdot \vec{b}:=\frac{1}{4}\left(|\vec{a}+\vec{b}|^{2}-|\vec{a}-\vec{b}|^{2}\right) .
\end{aligned}
$$

'?, por sua vez, tem as propriedades para ser chamado de um produto interno e foi inteiramente construído com um compasso. 
Um espaço vetorial munido com um produto interno gera naturalmente um espaço com norma pondo

$$
|\vec{a}|:=\sqrt{\vec{a} \cdot \vec{a}}
$$

Por outro lado, um espaço normado não precisa, necessariamente ter um produto interno. O enfoque operacional caminha em sentido contrário à estruturação usual na matemática. Primeiro constrói-se a norma (módulo) e só daí definimos o produto interno. Por fim, justificando a conexão com ângulos, temos o

Teorema 3 Dados $\vec{a}$ e $\vec{b}$ em $\mathbb{D}$,

$$
\vec{a} \cdot \vec{b}=|\vec{a}||\vec{b}| \cos [\Varangle(\vec{a}, \vec{b})] .
$$

Convidamos o leitor(a) a desenhar um par de vetores arbitrários e checar explicitamente esse resultado. Em particular, com $\vec{a}=\vec{b}$ o teorema se reduz a $|\vec{a}|^{2}=\vec{a} \cdot \vec{a}$. Dotamos assim $\mathbb{D}$ com um produto interno conectado com a norma.

Por praticidade, é costume construir bases em espaços vetoriais munidos com produto interno de modo que os elementos tenham todos módulo 1 e o produto interno entre elementos distintos seja nulo. Tais bases são chamadas de bases ortonormais. Consideremos a base $\mathcal{B}=\left\{\vec{a}_{1}, \vec{a}_{2}, \vec{a}_{3}\right\}$ de $\mathbb{D}$, de modo que qualquer vetor deslocamento $\vec{a}$ possa ser escrito unicamente como a combinação linear

$$
\vec{a}=\sum_{i=1}^{3} \alpha^{i} \vec{a}_{i} ; \alpha^{i} \in \mathbb{R}, i=1,2,3 .
$$

A partir de $\mathcal{B}$, construímos iterativamente o seguinte conjunto $\mathcal{B}^{\prime}=\left\{\vec{a}_{1}^{\prime}, \vec{a}_{2}^{\prime}, \vec{a}_{3}^{\prime}\right\}$ de elementos de $\mathbb{D}$, onde

$$
\begin{aligned}
& \vec{a}_{1}^{\prime}:=\vec{a}_{1} \\
& \vec{a}_{2}^{\prime}:=\quad \vec{a}_{2}-\frac{\left(\vec{a}_{2} \cdot \vec{a}_{1}^{\prime}\right)}{\left|\vec{a}_{1}^{\prime}\right|^{2}} \vec{a}_{1}^{\prime} \\
& \vec{a}_{3}^{\prime}:=\quad \vec{a}_{3}-\frac{\left(\vec{a}_{3} \cdot \vec{a}_{1}^{\prime}\right)}{\left|\vec{a}_{1}^{\prime}\right|^{2}} \vec{a}_{1}^{\prime}-\frac{\left(\vec{a}_{3} \cdot \vec{a}_{2}^{\prime}\right)}{\left|\vec{a}_{2}^{\prime}\right|^{2}} \vec{a}_{2}^{\prime} .
\end{aligned}
$$

Daí normalizamos os elementos de $\mathcal{B}^{\prime}$, gerando o conjunto $\left\{\hat{e}_{1}, \hat{e}_{2}, \hat{e}_{3}\right\}$, onde

$$
\hat{e}_{i}:=\frac{\vec{a}_{i}^{\prime}}{\left|\vec{a}_{i}^{\prime}\right|} ; i=1,2,3
$$

A passagem de $\mathcal{B}$ para $\left\{\hat{e}_{1}, \hat{e}_{2}, \hat{e}_{3}\right\}$ é conhecido como processo de ortonormalização de Gram-Schmidt [12]. Cada $\hat{e}_{i}$ não tem mais dimensão e neste sentido, geramos um espaço $\mathbb{U}$ com caráter universal no sentido de que qualquer grandeza física vetorial pode ser escrita a partir da combinação linear com valores de grandezas físicas unidimensionais $V_{G}$

$$
\vec{g}=\sum_{i=1}^{3} g^{i} \hat{e}_{i} ; g^{i} \in V_{G} ; i=1,2,3 .
$$

É exatamente essa construção acima que nos permite

(a) comparar direções de vetores que moram em espaços diferentes. Por exemplo: a resultante de força centrípeta sob uma partícula em movimento circular é perpendicular à velocidade.

(b) calcular o produto interno de vetores em espaços diferentes. Nossa definição de produto interno foi dada a partir de somas e diferenças, veja (38). Mas dadas grandezas vetoriais $\mathbb{G}$ e $\mathbb{H}$, escrevemos

$$
\begin{aligned}
\vec{g} \cdot \vec{h} & :=\left(\sum_{i=1}^{3} g^{i} \hat{e}_{i}\right) \cdot\left(\sum_{j=1}^{3} h^{j} \hat{e}_{j}\right) \\
& =\sum_{i=1}^{3} \sum_{j=1}^{3} g^{i} h^{j} \hat{e}_{i} \cdot \hat{e}_{j}=\sum_{i=1}^{3} g^{i} h^{i}
\end{aligned}
$$

O produto de termos do tipo $g^{i} h^{j}$ foi explicado com detalhes em [1]. Assim fica claro o significado, por exemplo, do trabalho de uma força $W=\vec{F} \cdot \vec{r}$.

Com isso, completamos a descrição sobre ângulos e seus desdobramentos ligados com grandezas vetoriais.

\section{Vetores duais ou co-vetores}

Nossa próxima grandeza física multidimensional é construída de maneira análoga a vetores como classes de pares ordenados de pontos. No lugar de pares de pontos, usaremos pares de retas paralelas. Esta nova grandeza poderá ser interpretada como funcionais lineares atuando sobre o espaço de vetores. Assim, dividiremos esta Seção em 3 partes. Na primeira, apresentaremos a nova grandeza física, que chamaremos de vetores duais ou co-vetores. Partimos então para a interpretação dos vetores duais como funcionais lineares sobre vetores e por fim, daremos um exemplo ilustrativo.

\subsection{Apresentando os vetores duais}

Para expor a construção, vamos considerar inicialmente determinado plano $\Pi \subset \mathcal{E}_{S R}$, de modo que $\left.\mathbb{D}\right|_{\Pi}$ é o espaço linear de vetores deslocamento que tem $\Pi$ como espaço afim. É um fato que $\left.\operatorname{dim} \mathbb{D}\right|_{\Pi}=2$. A generalização para 3 dimensões será feita posteriormente. Seja $\mathcal{R} \subset \Pi$ o conjunto de todas as retas contidas no plano $\Pi$. Seguindo a notação já adotada, os elementos de $\mathcal{R}$ serão denotados por $r$, eventualmente com índices abaixo: $r_{1}, r_{2}$, etc.

Consideremos agora o conjunto formado por pares de retas paralelas, que denotaremos por $\mathcal{P}_{(/, /)}$. Definimos a seguinte relação em $\mathcal{P}_{(/, /)} \times \mathcal{P}_{(/, /)}$,

$$
r_{1} / / r_{2} \sim_{\top} r_{3} / / r_{4}
$$

quando o par $r_{1} / / r_{2}$ alcança o par $r_{3} / / r_{4}$ por um transporte paralelo.

Naturalmente precisamos definir "alcançar" com o sentido dado acima, além do transporte paralelo de uma reta. O transporte paralelo de uma reta $r$ é feito da seguinte maneira. Tomamos pontos $A_{1}, A_{2}$ em $r$ e transportamos 
o vetor $\overrightarrow{\left(A_{1}, A_{2}\right)}$ até onde se deseja, chegando aos pontos, digamos, $A_{1}^{\prime}$ e $A_{2}^{\prime}$. Assim, diremos que a reta $r_{A_{1}^{\prime} A_{2}^{\prime}}$ definida por $A_{1}^{\prime}$ e $A_{2}^{\prime}$ corresponde ao transporte paralelo da reta $r$. Usaremos a notação $r_{\mathrm{T}}$ para indicar que a reta $r$ foi transportada. Por fim, dizemos que neste caso, a reta $r$ alcança $r_{A_{1}^{\prime} A_{2}^{\prime}}$. Agora, na relação 48, ' $r_{1} / / r_{2}$ alcança $r_{3} / / r_{4}$ ' significa que $r_{1}$ alcança $r_{3}$ e $r_{2}$ alcança $r_{4}$.

É um fato experimental que 48 define uma relação de equivalência, cuja demonstração pode ser obtida nos mesmos moldes do que aquela para vetores feita anteriormente.

Já estamos caminhando para a obtenção de uma grandeza física: temos o seu domínio, dado por pares de retas paralelas e uma relação de equivalência, que caracteriza objetos do mesmo tipo. Chamaremos as classes de equivalência em $\mathcal{P}_{(/, /)} / \sim_{T}$ de vetores duais ou co-vetores. Para completar a construção, precisamos ainda definir uma soma de classes em $\mathcal{P}_{(/, /)} / \sim_{T}$, a multiplicação por números reais e por fim, fornecer os valores às classes. Esta última etapa será um pouco diferente daquela para grandezas unidimensionais por uma razão simples. Os co-vetores serão interpretados como funcionais lineares sobre vetores.

Começamos com a soma. Gostaríamos de construir $\left[r_{1} / / r_{2}\right]+\left[r_{3} / / r_{4}\right]=\left[r_{5} / / r_{6}\right]$, onde $[r / / s]$ representa a classe gerada pelo par de retas paralelas $r$ e $s$. Gostaríamos também de mostrar que tal soma é de fato uma soma de classes, isto é, ela é independente dos elementos de $\left[r_{1} / / r_{2}\right]$ e $\left[r_{3} / / r_{4}\right]$ utilizados para se obter $\left[r_{5} / / r_{6}\right]$.

Sejam então $r_{1} / / r_{2}$ e $r_{3} / / r_{4}$ elementos das distintas classes $\left[r_{1} / / r_{2}\right]$ e $\left[r_{3} / / r_{4}\right]$. Dado um ponto $O \in \Pi$, faremos o seguinte:

1) Transportamos paralelamente o par $r_{1} / / r_{2}$ até que $r_{1}$ alcance $O$. Chamamos de $A_{1}$ a interseção de $\left(r_{1}\right)_{\top}$ com $r_{3}$ e $A_{2}$ a interseção de $\left(r_{2}\right)_{\mathrm{T}} \operatorname{com} r_{4}$. Tais interseções estão bem definidas pois as classes $\left[r_{1} / / r_{2}\right]$ e $\left[r_{3} / / r_{4}\right]$ são distintas.

2) Transportamos agora o par $\left[r_{3} / / r_{4}\right]$ até que $r_{3}$ alcance $O$. Assim como antes, $A_{3}=\left(r_{3}\right)_{\top} \cap r_{1}$ e $A_{4}=$ $\left(r_{4}\right)_{\mathrm{T}} \cap r_{2}$.

Consideremos as retas $r_{A_{1} A_{3}}$ e $r_{A_{2} A_{4}}$. Por construção, elas são paralelas. Definiremos então

$$
\left[r_{1} / / r_{2}\right]+\left[r_{3} / / r_{4}\right]:=\left[r_{A_{1} A_{3}} / / r_{A_{2} A_{4}}\right] .
$$

Mostrar a independência dos pares das classes escolhidos para construir a soma dada pelos passos 1 e 2 acima significa escolher pares $r_{1}^{\prime} / / r_{2}^{\prime} \in\left[r_{1} / / r_{2}\right]$ com $r_{1}^{\prime} / / r_{2}^{\prime} \neq$ $r_{1} / / r_{2}$ e $r_{3}^{\prime} / / r_{4}^{\prime} \in\left[r_{3} / / r_{4}\right] \operatorname{com} r_{3}^{\prime} / / r_{4}^{\prime} \neq r_{3} / / r_{4}$, além de um ponto $O^{\prime}$ arbitrário, construir $r_{A_{1}^{\prime} A_{3}^{\prime}} / / r_{A_{2}^{\prime} A_{4}^{\prime}}$ e constatar que existe um transporte paralelo tal que

$$
\left(r_{A_{1} A_{3}} / / r_{A_{2} A_{4}}\right)_{\mathrm{T}}=r_{A_{1}^{\prime} A_{3}^{\prime}} / / r_{A_{2}^{\prime} A_{4}^{\prime}} .
$$

A Figura 15 ilustra tal demonstração. As duplas setas $\leftrightharpoons$ em vermelho indicam o transporte paralelo de pares de retas.

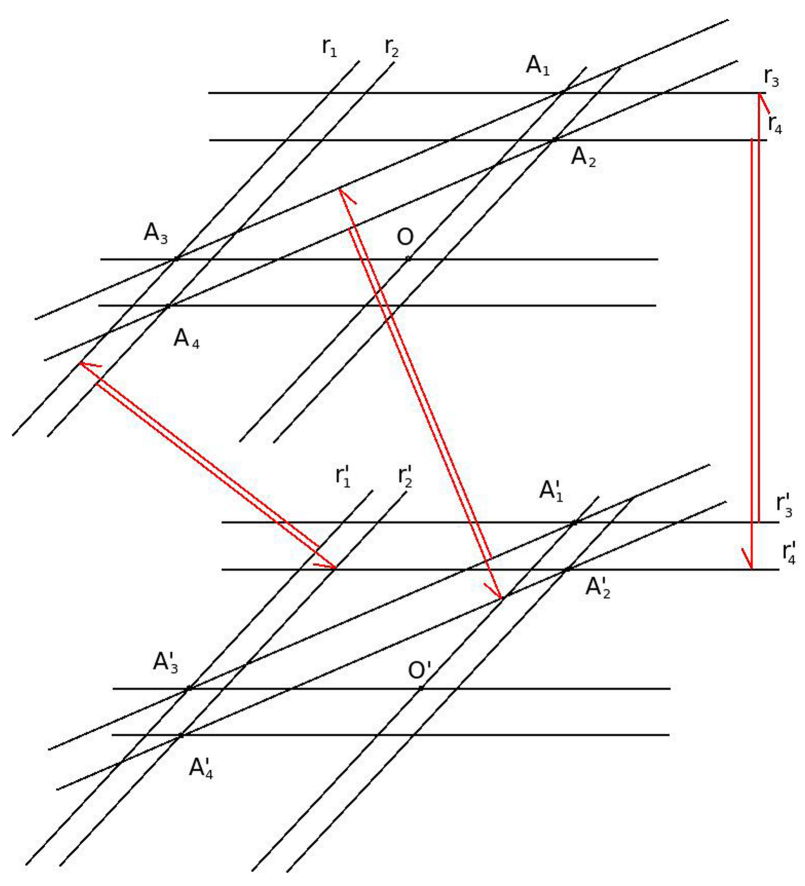

Figura 15: Representação da soma de classes de pares de retas.

A soma apresentada acima é patológica quando os pares $r_{1} / / r_{2}$ e $r_{3} / / r_{4}$ possuem retas paralelas, digamos, $r_{1}$ paralela a $r_{3}$. Esta dificuldade pode ser contornada escrevendo um dos pares como a soma de outros dois e, por fim, utilizando a associatividade da soma. A multiplicação por número é herdada da multiplicação de vetores por reais. Sejam $r_{1} / / r_{2} \in\left[r_{1} / / r_{2}\right]$ e $P_{1}$ e $P_{2}$ pontos arbitrários de $r_{1}$ e $r_{2}$, respectivamente. A multiplicação $\alpha \overrightarrow{\left(P_{1}, P_{2}\right)}$ de números por vetores já foi definida. Assim, considere

$$
\overrightarrow{\left(P_{1}, P_{\alpha}\right)}:=\alpha \overrightarrow{\left(P_{1}, P_{2}\right)} .
$$

Transportamos $r_{1}$ paralelamente até o ponto $P_{\alpha}$, obtendo a reta $r_{\alpha}$. Por construção, $r_{1}$ é paralela a $r_{\alpha}$ e assim definimos

$$
\alpha\left[r_{1} / / r_{2}\right]=\left[r_{1} / / r_{\alpha}\right]
$$

A representação geométrica da definição acima pode ser vista na Figura 16. Neste caso, meramente ilustrativo, $r_{1} / / r_{\alpha}$ indica multiplicação com $0<\alpha<1$ e $r_{1} / / r_{\beta}$ representa multiplicação por $\beta>1$.

A verificação experimental que (52) define produto por número sobre classes é direta. Dados $r_{1} / / r_{2}$ e $r_{1}^{\prime} / / r_{2}^{\prime}$ elementos de $\left[r_{1} / / r_{2}\right]$, tomamos pontos arbitrários $P 1$, $P_{2}, P_{1}^{\prime}$ e $P_{2}^{\prime}$ em $r_{1}, r_{2}, r_{1}^{\prime}$ e $r_{2}^{\prime}$. Construímos então $r_{1} / / r_{\alpha}$ e $r_{1}^{\prime} / / r_{\alpha}^{\prime}$ a partir da prescrição acima. Nota-se então que existe um transporte paralelo que conecta os pares $r_{1} / / r_{\alpha}$ e $r_{1}^{\prime} / / r_{\alpha}^{\prime}$, indicando que eles estão na mesma classe. $\mathrm{O}$ quadro geométrico desta comprovação está na Figura 17.

Com um pouco de trabalho direto, é possível verificar que $\left(\mathcal{P}_{(/, /)} / \sim_{\mathrm{T}},+, \cdot\right)$, onde $+\mathrm{e} \cdot$ representam as operações 


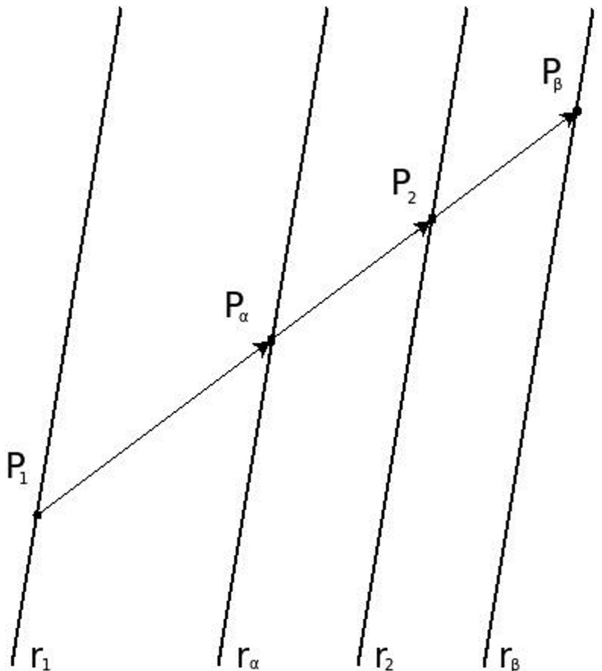

Figura 16: Definição do produto por números em $\mathcal{P}_{(/, /)} / \sim_{T}$.

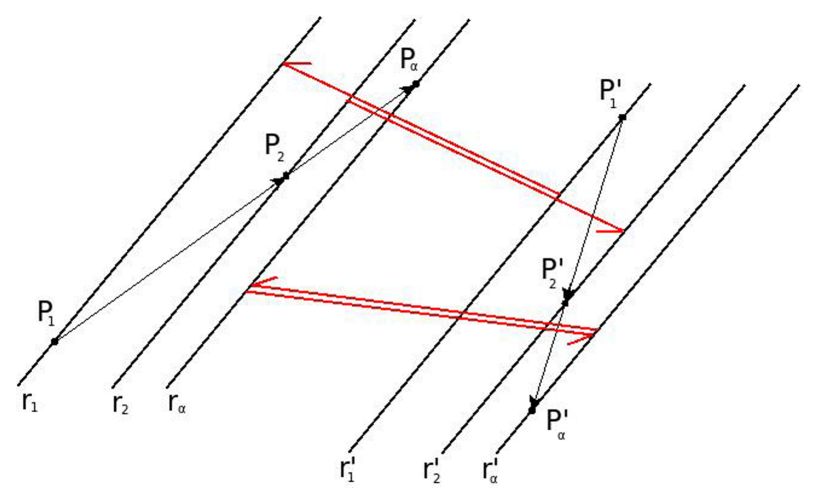

Figura 17: Comprovação experimental que (52) define o produto de classes por números.

de soma e produto por número, forma um espaço vetorial. Vamos denotar este espaço por $\left.\overline{\overline{\mathbb{D}}}\right|_{\Pi}$. Esta definição na nomenclatura tem duas razões. A primeira é que a dupla barra sobre $\mathbb{D}$ nos lembra que temos um conjunto de classes de retas paralelas. A segunda, talvez mais importante, é que podemos interpretar elementos deste nosso novo espaço vetorial como operadores lineares que atuam sobre $\left.\mathbb{D}\right|_{\Pi}$ fornecendo como resposta valores da grandeza física distância, a partir de projeções. Os detalhes serão dados a seguir.

\subsection{Interpretando $\left.\overline{\overline{\mathbb{D}}}\right|_{\Pi \text { como espaço de }}$ funcionais lineares sobre $\left.\mathbb{D}\right|_{\Pi}$}

A construção de $\mathbb{D}$ como pares de pontos que se conectam por um transporte paralelo é bastante profunda, mas pouco prática. Neste caso, o que se faz é fixar um ponto $O \in \mathcal{E}_{S R}$, fixar certas coordenadas, digamos, cartesianas, de modo que qualquer ponto $P$ de $\mathcal{E}_{S R}$ é representado de forma única pela ponta do vetor

$$
\overrightarrow{(O, P)}=\left(x_{P}-x_{0}\right) \hat{i}+\left(y_{P}-y_{O}\right) \hat{j}+\left(z_{P}-z_{O}\right) \hat{k}
$$

com $x, y$ e $z$ mapeamentos diferenciáveis que levam pontos de $\mathcal{E}_{S R}$ em valores de distância e $\{\hat{i}, \hat{j}, \hat{k}\}$ é uma base ortonormal de U. A realização de $\left.\overline{\overline{\mathbb{D}}}\right|_{\Pi}$ é feita de forma análoga. Fixamos inicialmente um ponto $O \mathrm{em}$ $\mathcal{E}_{S R}$ de modo que qualquer ponto $P$ de $\Pi$ seja representado unicamente em coordenadas cartesianas por $\vec{r}(P)=x_{P} \hat{i}+y_{P} \hat{j}=\overrightarrow{(O, P)}$. Por construção, $O$ é o que chamamos de origem do sistema de coordenadas. Desta maneira, temos um par de retas, a saber, os eixos coordenados $x$ e $y$ a partir dos quais construiremos $\left.\overline{\overline{\mathbb{D}}}\right|_{\Pi \text {. Fixado }}$ $\left.\vec{r}(P) \in \mathbb{D}\right|_{\Pi}$, denotaremos $d x[\cdot]$ o elemento de $\left.\overline{\overline{\mathbb{D}}}\right|_{\Pi}$ que representa o par de retas paralelas $x=0$ e $x=x_{P}$, que projeta $\vec{r}(P)$ no eixo $x$, isto é, $d x[\vec{r}(P)]=x_{P}$. Da mesma maneira, na direção $y$, e escrevemos $d y[\vec{r}(P)]=y_{P}$. A Figura 18 fornece uma representação geométrica da atuação de $d x[\cdot]$ e $d y[\cdot]$ sobre $\left.\mathbb{D}\right|_{\Pi}$. Vamos enumerar uma lista de propriedades das projeções assim construídas.

1) A projeção $d x[\cdot]$ é um operador linear que leva vetores em valores de distância

$$
\begin{aligned}
d x[\cdot]:\left.\mathbb{D}\right|_{\Pi} & \rightarrow V_{L} \\
\vec{r} & \mapsto d x[\vec{r}]=r_{x},
\end{aligned}
$$

onde $\vec{r}=r_{x} \hat{i}+r_{y} \hat{j}$. A notação $d x[\cdot]$ indica que temos um mapeamento, onde no lugar do ponto colocamos um vetor, obtendo como resposta uma distância.

A linearidade é obtida diretamente. Dados $\vec{a}$ e $\vec{b}$ com componentes $a_{x}, a_{y}, b_{x}$ e $b_{y}$ e $\alpha \in \mathbb{R}$,

$$
\begin{aligned}
d x[\alpha \vec{a}+\vec{b}] & =\alpha a_{x}+b_{x} \\
& =\alpha d x[\vec{a}]+d x[\vec{b}] .
\end{aligned}
$$

Por construção, um valor de distância é não-negativo. Por outro lado, o conjunto de valores de distância $\left(V_{L},+, \cdot\right)$ é um espaço vetorial unidimensional sobre o corpo dos reais [1]. Assim, dado $d \in V_{L}, \alpha d \in V_{L}, \forall \alpha \in \mathbb{R}$. Em particular, $-d \in V_{L}$. Apesar de soar inconsistente, podemos interpretar uma distância negativa como, por exemplo, a coordenada cartesiana de um vetor que aponta no sentido negativo do eixo $x$.

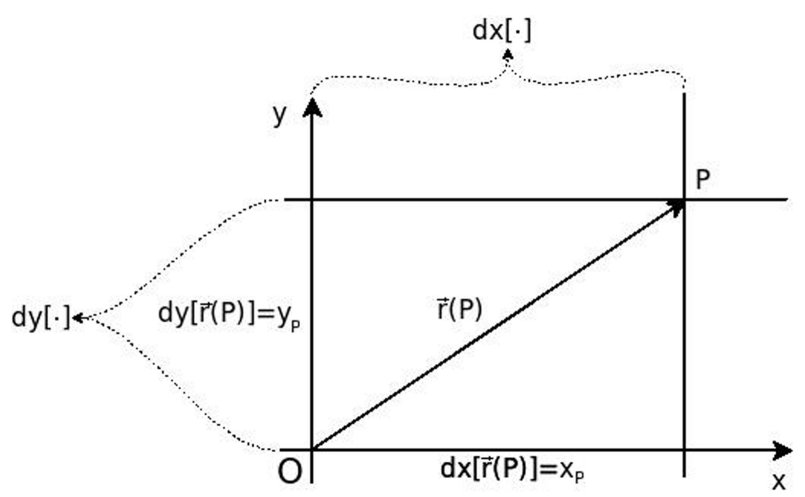

Figura 18: Pares de retas paralelas $(d x[\cdot]$ e $d y[\cdot])$ atuando sobre o vetor $\vec{r}(P)$. 
2) A expressão 55 permite interpretar a multiplicação por escalar em $\left.\overline{\overline{\mathbb{D}}}\right|_{\Pi}$ de maneira consistente com o que fizemos antes,

$$
\alpha d x[\vec{a}]=d x[\alpha \vec{a}]=\alpha a_{x} .
$$

As duas propriedades acima são naturalmente válidas também para $d y[\cdot]$. Passamos agora à terceira e última.

3) Nosso objetivo é agora interpretar geometricamente

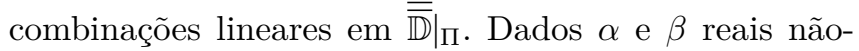
nulos, gostaríamos de dar significado para $\alpha d x[\cdot]+\beta d y[\cdot]$. Definimos, inicialmente, a soma de operadores lineares de forma usual e usamos (56),

$$
\begin{aligned}
(\alpha d x[\cdot]+\beta d y[\cdot])(\vec{a}): & :=\alpha d x[\vec{a}]+\beta d y[\vec{a}] \\
& =\alpha a_{x}+\beta a_{y} .
\end{aligned}
$$

Ora, (57) corresponde a uma expressão bem conhecida. Ela é o produto interno entre os vetores $\alpha \hat{i}+\beta \hat{j} \in \mathbb{R}^{2} \mathrm{e}$ $\vec{a}=a_{x} \hat{i}+\left.a_{y} \hat{j} \in \mathbb{D}\right|_{\Pi}$. Lembremos que mesmo morando em espaços diferentes, tal produto é bem definido, veja o comentário no fim da Seção 4 . Como vimos

$$
\alpha a_{x}+\beta a_{y}=\sqrt{\alpha^{2}+\beta^{2}}|\vec{a}| \cos \Varangle[(\alpha, \beta), \vec{a}] .
$$

Esta expressão corresponde à projeção do vetor $\vec{a}$ na reta que suporta o vetor $(\alpha, \beta)$. Em termos de projeções, (57) pode ser vista então como a combinação linear

$$
\alpha r_{1} / / r_{2}+\beta r_{3} / / r_{4}
$$

onde $r_{1}$ corresponde à reta $x=0$ e $r_{2}$ à reta com equação $x=\frac{1}{\alpha}\left(\alpha a_{x}+\beta a_{y}\right)$. Já $r_{3}$ e $r_{4}$ são as retas com equações $y=0$ e $y=\frac{1}{\beta}\left(\alpha a_{x}+\beta a_{y}\right)$, de modo que a soma indicada por 59 fornece o par de retas paralelas com uma delas passando pela origem e a outra tocando a ponta do vetor $\vec{a}$, sendo que a inclinação deste par, que chamaremos de $r_{5} / / r_{6}$ é dado por $-\frac{\alpha}{\beta}$. Logo, $\vec{a}$ é projetado ortogonalmente à reta que suporta o vetor $\alpha \hat{i}+\beta \hat{j}$ e o resultado corresponde à tal projeção multiplicado por um fator de escala, que é a norma de $\alpha \hat{i}+\beta \hat{j}$. Supondo o vetor $\alpha \hat{i}+\beta \hat{j}$ unitário, representamos a interpretação dada acima na Figura 19

Nossos argumentos anteriores mostram um resultado bastante geral: qualquer operador linear que leva vetores deslocamento em valores de distância pode ser representado unicamente por um produto interno com vetor fixo de $\mathbb{R}^{2} \cdot\{d x[\cdot], d y[\cdot]\}$ forma uma base do espaço bidimensional $\left.\overline{\overline{\mathbb{D}}}\right|_{\Pi}$, isto é, qualquer elemento deste espaço tem a forma $\alpha d x[\cdot]+\beta d y[\cdot]$. Em outras palavras, existe uma bijeção de $\left.\overline{\overline{\mathbb{D}}}\right|_{\Pi}$ com o conjunto de operadores lineares que levam vetores em valores de distância, denotado por $\mathcal{L}\left(\left.\mathbb{D}\right|_{\Pi}, V_{L}\right)$. Há duas maneiras para representarmos os elementos de $\mathcal{L}\left(\left.\mathbb{D}\right|_{\Pi}, V_{L}\right)$. A primeira, montamos a matriz de representação de $\alpha d x[\cdot]+\beta d y[\cdot]$, que é dada pela linha $\left(\begin{array}{ll}\alpha & \beta\end{array}\right)$. Assim, representando $\vec{a}$ pela coluna com

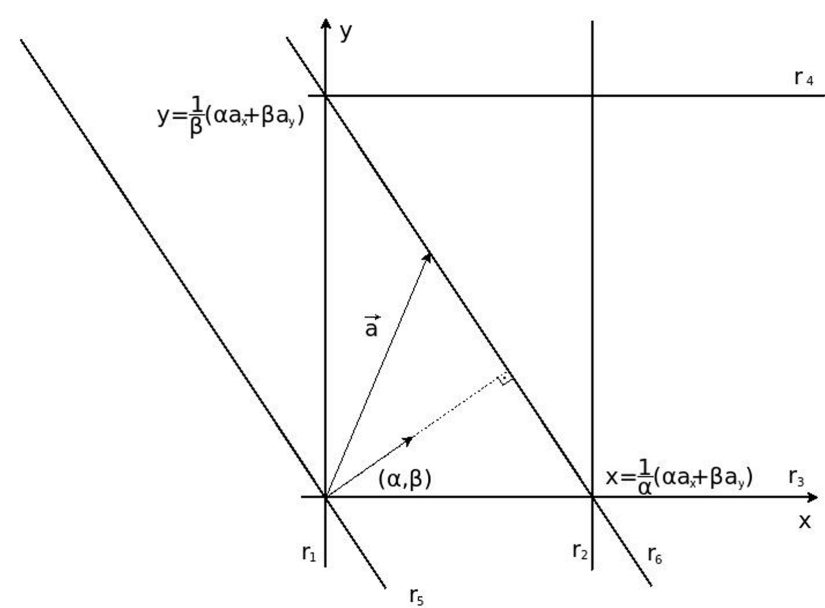

Figura 19: Projeção ortogonal de $\vec{a}$ (representada pelo segmento tracejado) pela atuação de $\alpha r_{1} / / r_{2}+\beta r_{3} / / r_{4}=r_{5} / / r_{6}$, com $\alpha^{2}+\beta^{2}=1$.

entradas $a_{x}$ e $a_{y}$, ficamos com

$$
(\alpha d x[\cdot]+\beta d y[\cdot])(\vec{a})=\left(\begin{array}{ll}
\alpha & \beta
\end{array}\right)\left(\begin{array}{l}
a_{x} \\
a_{y}
\end{array}\right)=\alpha a_{x}+\beta a_{y} .
$$

A segunda maneira é representar o elemento de $\left.\overline{\overline{\mathbb{D}}}\right|_{\Pi}$ por um produto interno

$$
\alpha d x[\cdot]+\beta d y[\cdot]=(\alpha, \beta) \cdot[\cdot] .
$$

O leitor com conhecimento de análise funcional reconhece na expressão acima o Teorema de Representação de Riesz 11, 13, 14.

A generalização para três dimensões é direta. $\{d x[\cdot]$, $d y[\cdot], d z[\cdot]\}$ é base do espaço tridimensional $\overline{\overline{\mathbb{D}}} . d x[\cdot]$, $d y[\cdot]$ e $d z[\cdot]$ são pares de planos paralelos, cujo o primeiro elemento do par corresponde a $y z, x z$ e $x y$ respectivamente. $\mathrm{O}$ segundo elemento por sua vez toca a ponta do vetor $\vec{a} \in \mathbb{D}$ sobre o qual as projeções atuam, de modo que

$$
(\alpha d x[\cdot]+\beta d y[\cdot]+\gamma d z[\cdot])(\vec{a})=\alpha a_{x}+\beta a_{y}+\gamma a_{z}
$$

onde $\vec{a}=\left(a_{x}, a_{y}, a_{z}\right)$ e $\alpha, \beta$ e $\gamma$ são reais. Assim como antes, qualquer elemento de $\overline{\overline{\mathbb{D}}}$ pode ser representado ou pela linha $\left(\begin{array}{lll}\alpha & \beta & \gamma\end{array}\right)$ atuando de maneira linear sobre as componentes de um vetor de $\mathbb{D}$ ou como o operador linear $(\alpha, \beta, \gamma) \cdot[\cdot]$. Em ambos os casos, vetores são levados linearmente em valores de distância. Com isso, caracterizamos o isomorfismo

$$
\overline{\overline{\mathbb{D}}} \longleftrightarrow \mathcal{L}\left(\mathbb{D}, V_{L}\right)
$$

Assim como antes, $\alpha \hat{i}+\beta \hat{j}+\gamma \hat{k}$ é o vetor normal ao plano que projeta o vetor deslocamento. A representação desta interpretação é dada na Figura 20, onde também consideramos $\sqrt{\alpha^{2}+\beta^{2}+\gamma^{2}}=1$. 


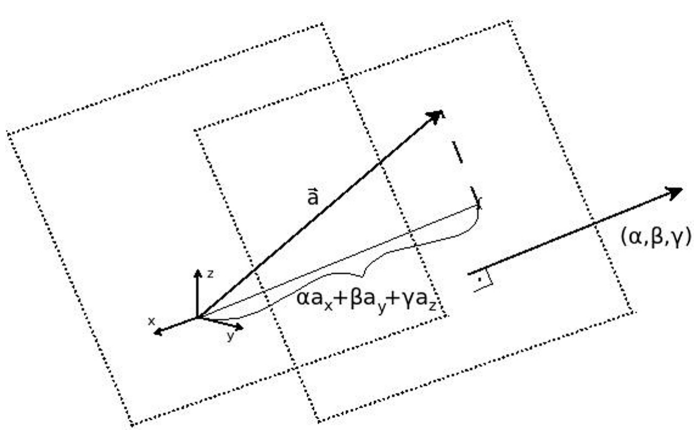

Figura 20: Projeção de um vetor por planos paralelos cujo vetor normal unitário é $(\alpha, \beta, \gamma)$.

Completamos assim nossa construção sobre co-vetores. Na próxima subseção discutiremos um exemplo desta construção, mais usual do que se imagina. Antes contudo, façamos um breve comentário sobre notação. Uma vez escolhidos eixos coordenados $x, y$ e $z$, já escolhemos um dos planos de projeção que compõem nossos co-vetores. Assim, no lugar de usar duas barras sobre $\mathbb{D}$, usaremos somente uma, $\overline{\mathbb{D}}$. Pela mesma razão, cada co-vetor será representado por $\bar{p}$, para lembrar de projeção, sendo que $\left\{\overline{d x}^{i}[\cdot] ; i=1,2,3\right\}$ representa uma base de $\overline{\mathbb{D}}$.

\subsection{Exemplo de co-vetores: vetor de onda}

Toda construção feita anteriormente tem forte caráter geométrico e parece estar muito próxima de um matemático. Contudo, todos que estudam oscilações e ondas se deparam com ela. Consideremos assim, um exemplo ilustrativo. Em uma cuba com água, geramos ondas planas, que se deslocam com velocidade $\vec{V}$ bem definida. Tal setup experimental pode ser montado com um kit da Cidepe 15. e projetado na parede como mostram as imagens na Figura 21.

Podemos nos perguntar qual é a amplitude da onda em determinado ponto do espaço. A resposta é dada por um mapeamento que leva pontos do espaço (mapeados com um sistema de coordenadas) em determinado instante de
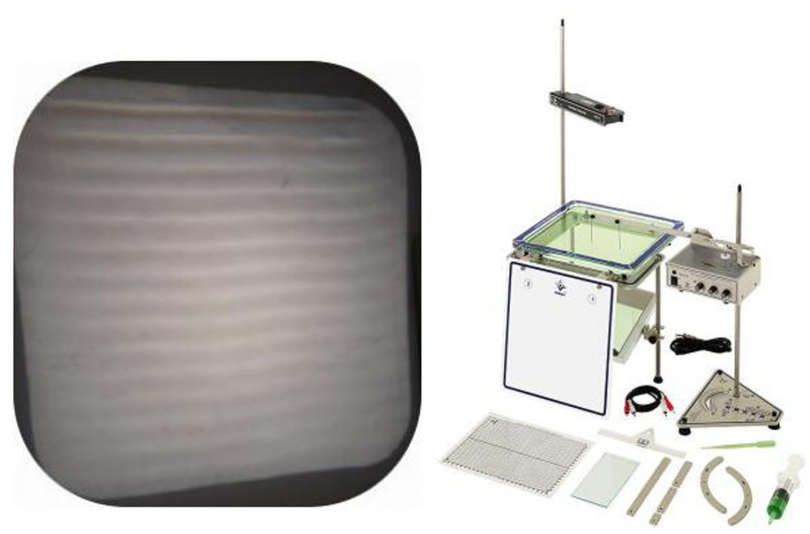

Figura 21: Projeção de ondas planas (arquivo dos autores) gerada por um kit da Cidepe (retirado do sítio [15]). tempo em valores de distância

$$
\begin{aligned}
\psi: \mathbb{D} \times V_{T} & \rightarrow V_{L} \\
(\vec{r}, t) & \mapsto \psi(\vec{r}, t) .
\end{aligned}
$$

$\psi$ é chamado de perfil (ou função) da onda. A onda gerada na Figura 21 é bem comportada e podemos usar um referencial privilegiado, $\mathcal{R}^{\prime \prime}$ que se desloca junto com a onda, digamos em uma direção $x^{\prime \prime}$. Neste referencial, a onda tem um perfil estático, logo,

$$
\psi\left(x^{\prime \prime}, y^{\prime \prime}, z^{\prime \prime}, t\right)=A \cos \left(k x^{\prime \prime}\right)
$$

onde $A$ é a amplitude da onda. Consideremos agora outro referencial, $\mathcal{R}^{\prime}$, cujo eixo $x^{\prime}$ está na direção de $\vec{V}$. Neste caso, $\mathcal{R}^{\prime}$ e $\mathcal{R}^{\prime \prime}$ têm coordenadas que se conectam com

$$
x^{\prime \prime}=x^{\prime}-V t,
$$

onde $V=|\vec{V}|$. O fator multiplicativo $k$ que aparece em (65) garante que $k x^{\prime \prime}$ é adimensional, uma vez que a função cosseno leva números em números.

Para dar um caráter mais geral, no sentido de descrever o perfil $\psi$ em um referencial mais geral, trocamos finalmente a descrição da onda para um referencial $\mathcal{R}$ rotacionado em relação a $\mathcal{R}^{\prime}$ por um ângulo $\xi=\operatorname{arc} \operatorname{tg}\left(\frac{V_{x}}{V_{y}}\right)$ onde $V_{x}$ e $V_{y}$ são as componentes da velocidade da onda vista por um observador em $\mathcal{R}$. Nesse caso,

$$
\left(\begin{array}{l}
x^{\prime} \\
y^{\prime} \\
z^{\prime}
\end{array}\right)=\left(\begin{array}{ccc}
\cos \xi & \operatorname{sen} \xi & 0 \\
-\operatorname{sen} \xi & \cos \xi & 0 \\
0 & 0 & 1
\end{array}\right)\left(\begin{array}{l}
x \\
y \\
z
\end{array}\right) .
$$

Por fim, utilizando as trocas entre referenciais de $\mathcal{R}^{\prime \prime}$ para $\mathcal{R}^{\prime}$ e de $\mathcal{R}^{\prime}$ para $\mathcal{R}$, substituídas de volta em (65), encontramos o perfil da onda visto por um observador em $\mathcal{R}$

$$
\psi(x, y, z, t)=A \cos (k \cos \xi x+k \operatorname{sen} \xi y-V t) .
$$

Representamos a troca de referenciais e a onda plana na Figura 22

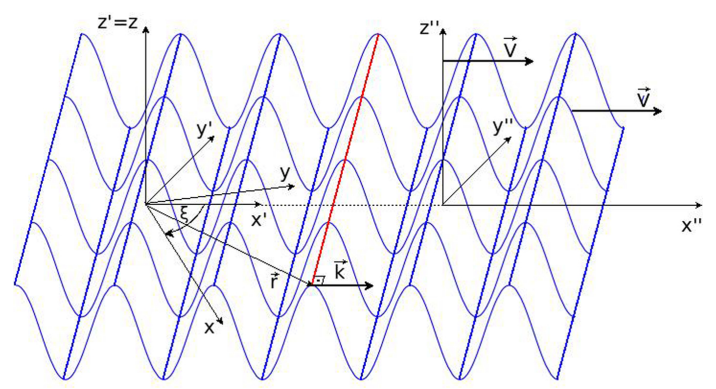

Figura 22: Referenciais utilizados para obtenção do perfil da onda plana $\psi$ no referencial $\mathcal{R}$. 
A parte espacial do argumento em (68) agora já nos é conhecida

$$
k \cos \xi x+k \operatorname{sen} \xi y=(k \cos \xi, k \operatorname{sen} \xi, 0) \cdot(x, y, z) .
$$

$\mathrm{O}$ vetor $\vec{k}=(k \cos \xi, k \operatorname{sen} \xi, 0)$ é conhecido como vetor de onda e fornece a direção de propagação, além de carregar também informação sobre a periodicidade espacial da onda plana. Mais ainda, $\vec{k} \cdot[\cdot]$ é um operador linear que leva vetores em números. Ele é perpendicular às retas paralelas definidas por uma frente de onda, tão visível na Figura 21 e consistente com nossa interpretação geométrica prévia para co-vetores. O leitor atento notaria que escolhemos uma direção para a propagação da onda no referencial $\mathcal{R}$ sem componente $z$. A generalização contudo é direta. No lugar da expressão (67), teríamos uma matriz com ângulos de Euler, que conectaria $\mathcal{R}$ e $\mathcal{R}^{\prime}$, de forma que $\vec{k}$ tivesse componentes nas 3 direções, mantendo todo o resto da interpretação inalterada.

\section{Tensores}

Para caracterizar nossa próxima grandeza multidimensional, precisamos levar nossa abstração um pouco mais longe, sem perder contudo, contato com nossa prescrição operacional. Como vimos anteriormente com vetores duais, a própria grandeza física era um mapeamento. Assim como antes, a solução para construir grandezas físicas multidimensionais reside em um tipo de mapeamento bem específico: os lineares. Para motivar o uso de tais mapeamentos, suponha que você vá em uma loja de departamento para comprar corda. O vendedor prontamente tem uma máquina que transforma seu pedido em metros para reais. Este operador, que chamaremos de $\mu$, tem como domínio o espaço de valores de distância $V_{L}$ e como contradomínio, nossa moeda.

$$
\mu: V_{L} \rightarrow \$
$$

O mapeamento $\mu$ tem algumas propriedades interessantes. A primeira é que comprar dois pedaços de tamanhos $l_{1}$ e $l_{2}$, fornece o mesmo valor que um pedaço de tamanho $l_{1}+l_{2}$, isto é,

$$
\mu\left(l_{1}+l_{2}\right)=\mu\left(l_{1}\right)+\mu\left(l_{2}\right)
$$

Afinal, não queremos nem levar prejuízo e tampouco dar prejuízo à loja (principalmente em tempos de crise), e estas situações ocorrem justamente quando, $\mu\left(l_{1}+l_{2}\right) \neq$ $\mu\left(l_{1}\right)+\mu\left(l_{2}\right)$. Naturalmente, podemos multiplicar nosso tamanho da corda por $r \in \mathbb{R}$ e esperamos que o preço sofra a mesma contração $(r<1)$ ou dilatação $(r>1)$. Assim,

$$
\mu(r l)=r \mu(l)
$$

Nosso $\mu$ tem a mesma estrutura que as densidades de massa tão conhecidas: elas são operadores lineares que le- vam tamanhos (comprimento, área e volume) em valores de massa de maneira linear, sendo o próprio mapeamento visto como a grandeza física. Com este exemplo em mãos, vamos estruturar certos mapeamentos lineares em conjuntos que contenham vetores e co-vetores. Começamos interpretando o próprio $\mathbb{D}$ como o espaço de operadores lineares sobre $\overline{\mathbb{D}}$. Dados $\vec{a} \in \mathbb{D}$ e $\bar{p} \in \overline{\mathbb{D}}$, definimos o seguinte operador linear,

$$
\begin{aligned}
\vec{a}: \overline{\mathbb{D}} & \rightarrow V_{L} \\
\bar{p} & \mapsto \vec{a}(\bar{p}):=\bar{p}(\vec{a}) \in V_{L}
\end{aligned}
$$

De acordo com a Seção $5, \bar{p}(\vec{a})$ nada mais é que a projeção do vetor $\vec{a}$. A linearidade é imediata,

$$
\begin{aligned}
\vec{a}\left(\bar{p}_{1}+r \bar{p}_{2}\right) & =\left(\bar{p}_{1}+r \bar{p}_{2}\right)(\vec{a})= \\
=\bar{p}_{1}(\vec{a})+r \bar{p}_{2}(\vec{a}) & =\vec{a}\left(\bar{p}_{1}\right)+r \vec{a}\left(\bar{p}_{2}\right) .
\end{aligned}
$$

Interpretando o próprio $\mathbb{D}$ como um espaço de operadores lineares, definimos agora

Definição 9 O produto tensorial de $\mathbb{D}$ por $\mathbb{D}$, denotado pelo símbolo $\mathbb{D} \otimes \mathbb{D}$, é o conjunto formado por todas as aplicações bilineares de $\overline{\mathbb{D}} \times \overline{\mathbb{D}}$ em $V_{G}$. Os elementos de $\mathbb{D} \otimes \mathbb{D}$ serão denotados $\vec{a} \otimes \vec{b}$ e atuam no par $(\bar{p}, \bar{q}) \in \overline{\mathbb{D}} \times \overline{\mathbb{D}}$ da seguinte maneira,

$$
(\vec{a} \otimes \vec{b})(\bar{p}, \bar{q}):=\bar{p}(\vec{a}) \bar{q}(\vec{b}) .
$$

A linearidade em cada entrada é consequência de (74).

Como o produto cartesiano de espaços vetoriais é um espaço vetorial, o conjunto de operadores lineares sobre tal espaço, $\mathbb{D} \otimes \mathbb{D}$, também tem estrutura de espaço vetorial. Com (75) podemos ainda verificar que $\otimes$ tem as propriedades usuais de um produto, a saber

1. $\left(\alpha \vec{a}_{1}\right) \otimes \vec{a}_{2}=\vec{a}_{1} \otimes\left(\alpha \vec{a}_{2}\right)$.

2. $\vec{a}_{1} \otimes\left(\vec{a}_{2}+\vec{a}_{3}\right)=\vec{a}_{1} \otimes \vec{a}_{2}+\vec{a}_{1} \otimes \vec{a}_{3}$.

3. $\left(\vec{a}_{1}+\vec{a}_{2}\right) \otimes \vec{a}_{3}=\vec{a}_{1} \otimes \vec{a}_{3}+\vec{a}_{2} \otimes \vec{a}_{3}$.

Por fim, $\vec{a}_{1} \otimes \vec{a}_{2}+\vec{a}_{3} \otimes \vec{a}_{4}$ nada mais é que a soma de dois operadores lineares atuando da maneira usual

$$
\begin{array}{r}
\left(\vec{a}_{1} \otimes \vec{a}_{2}+\vec{a}_{3} \otimes \vec{a}_{4}\right)(\bar{p}, \bar{q})= \\
=\left(\vec{a}_{1} \otimes \vec{a}_{2}\right)(\bar{p}, \bar{q})+\left(\vec{a}_{3} \otimes \vec{a}_{4}\right)(\bar{p}, \bar{q})
\end{array}
$$

Tal soma é desejável pois, como discutido anteriormente, são os próprios mapeamentos que serão nossas grandezas multidimensionais. Por fim, com $\otimes$ podemos escrever uma base de $\mathbb{D} \otimes \mathbb{D}$. Ela é dada por $\left\{\vec{a}_{i} \otimes \vec{a}_{j} \mid i, j=1,2,3\right\}$ com $\vec{a}_{1}, \vec{a}_{2}, \vec{a}_{3}$ base de $\mathbb{D}$. Toda a formulação anterior pode ser repetida com a troca de $\mathbb{D}$ por $\overline{\mathbb{D}}$, isto é, um elemento $\bar{p} \otimes \bar{q}$ de $\overline{\mathbb{D}} \otimes \overline{\mathbb{D}}$ é um operador bilinear

$$
\bar{p} \otimes \bar{q}: \mathbb{D} \times \mathbb{D} \rightarrow V_{G} .
$$

Podemos ainda ir mais longe e definir um conjunto que chamaremos espaço de tensores do tipo $(r, s)$ com $r, s \in$ $\mathbb{N} \cup\{0\}$ 
Definição $10 T_{s}^{r}(\mathbb{D})=\mathbb{D} \otimes \cdots \otimes \mathbb{D} \otimes \overline{\mathbb{D}} \otimes \cdots \otimes \overline{\mathbb{D}}:=\otimes_{r} \mathbb{D} \otimes_{s} \overline{\mathbb{D}}$ é o conjunto onde cada elemento l de $T_{s}^{r}(\mathbb{D})$ é dado por uma aplicação multilinear

$$
l: \overline{\mathbb{D}} \times \cdots \times \overline{\mathbb{D}} \times \mathbb{D} \times \cdots \times \mathbb{D} \rightarrow V_{G} .
$$

$V_{G}$ é o espaço de valores de alguma grandeza unidimensional.

Ou seja, o espaço de tensores do tipo $(r, s)$ é o conjunto de todas as aplicações lineares que atuam no produto cartesiano $\times_{r} \overline{\mathbb{D}} \times_{s} \mathbb{D}$. Lembrando que há uma dualidade entre $\mathbb{D}$ e $\overline{\mathbb{D}}$ no sentido de que um pode ser visto como espaço de funcionais sobre o outro, então não é surpresa a ordem trocada em que $\mathbb{D}$ e $\overline{\mathbb{D}}$ aparecem nas expressões da Definição 10 e no domínio dos operadores $l \in \otimes_{r} \mathbb{D} \otimes_{s} \overline{\mathbb{D}}$. Para $r=s=0$, definimos $T_{0}^{0}:=V_{G}$.

Como discutido, $\otimes_{r} \mathbb{D} \otimes_{s} \overline{\mathbb{D}}$ tem estrutura de espaço vetorial. Fixando $\left\{\vec{a}_{1}, \vec{a}_{2}, \vec{a}_{3}\right\}$ uma base de $\mathbb{D}$ e $\left\{\bar{p}_{1}, \bar{p}_{2}, \bar{p}_{3}\right\}$ uma base de $\overline{\mathbb{D}}$, podemos prontamente escrever uma base $\mathcal{B}$ de $T_{s}^{r}(\mathbb{D})$ :

$$
\mathcal{B}=\left\{\vec{a}_{i_{1}} \otimes \vec{a}_{i_{2}} \otimes \cdots \otimes \vec{a}_{i_{r}} \otimes \bar{p}_{j_{1}} \otimes \cdots \otimes \bar{p}_{j_{s}}\right\},
$$

onde cada $i_{1}, \ldots, i_{r}, j_{1}, \ldots, j_{s}$ corre valores 1,2 e 3 .

Fixando coeficientes distintos para montar combinações lineares com a base $\mathcal{B}$ em $(79)$, teremos diferentes exemplos de tensores de interesse prático, que passamos a detalhar.

Exemplo 1:

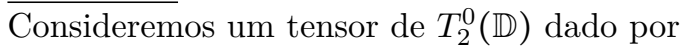

$$
\Pi(\cdot, \cdot)=\delta_{i j} \overline{d x}^{i}[\cdot] \otimes \overline{d x}^{j}[\cdot]: \mathbb{D} \times \mathbb{D} \rightarrow V_{L^{2}}
$$

onde $\delta_{i j}$ é o delta de Kroenecker, isto é, $\delta_{i j}=1$ quando $i=j$ e zero, caso contrário.

Aplicando $\Pi(\cdot, \cdot)$ a um par de vetores,

$$
\Pi(\vec{a}, \vec{b})=\delta_{i j} \overline{d x}^{i}[\vec{a}] \otimes \overline{d x}^{j}[\vec{b}]=\delta_{i j} a^{i} b^{j} .
$$

Um resultado bastante curioso, associado com este primeiro exemplo é o seguinte

Teorema $4 \Pi(\cdot, \cdot)$ define um produto interno em $\mathbb{D} e$ $\Pi(\vec{a}, \vec{b})=\delta_{i j} a^{i} b^{j}=\frac{1}{4}\left(|\vec{a}+\vec{b}|^{2}-|\vec{a}-\vec{b}|^{2}\right)=|\vec{a}||\vec{b}| \cos [\Varangle(\vec{a}, \vec{b})]$.

A demonstração é deixada ao cargo do leitor interessado. Esta é uma boa tarefa experimental em que temos que desenhar $\vec{a}, \vec{b}, \vec{a}+\vec{b}, \vec{a}-\vec{b}$, medir seus módulos, determinar $\Varangle(\vec{a}, \vec{b})$ ou o próprio cosseno diretamente. Notamos aqui a conexão entre o produto interno induzido por um tensor e o Teorema 3 .

Exemplo 2:

Vamos agora restringir $\mathbb{D}$ a um plano $\Pi$, como fizemos na Seção 4 Fixamos um sistema cartesiano de coordenadas e consideramos o seguinte elemento de $T_{2}^{0}\left(\left.\mathbb{D}\right|_{\Pi}\right)$,

$$
A(\cdot, \cdot):\left.\mathbb{D}\right|_{\Pi} \times\left.\mathbb{D}\right|_{\Pi} \rightarrow V_{L^{2}},
$$

$\operatorname{com} A(\cdot, \cdot)=\left|\epsilon_{a b} \overline{d x}^{a}[\cdot] \otimes \overline{d x}^{b}[\cdot]\right| . \epsilon_{a b}$ é o símbolo de LeviCivita em duas dimensões [16], isto é, $\epsilon_{12}=-\epsilon_{21}=1$, $\epsilon_{11}=\epsilon_{22}=0$. Assim, dados $\vec{a},\left.b \in \mathbb{D}\right|_{\Pi}$ temos,

$$
A(\vec{a}, \vec{b})=\left|\epsilon_{a b} \overline{d x}^{a}[\vec{a}] \otimes \overline{d x}^{b}[\vec{b}]\right|=\left|a^{1} b^{2}-a^{2} b^{1}\right| .
$$

Esta expressão também já conhecida é correspondente à área do paralelogramo definida pelo par $(\vec{a}, \vec{b})$. Neste caso, o mapeamento $A(\cdot, \cdot)$ divide o conjunto de pares de vetores em classes, tal que os pares geram paralelogramos com mesma área,

$$
(\vec{a}, \vec{b}) \sim_{A}(\vec{c}, \vec{d}) \Longleftrightarrow A(\vec{a}, \vec{b})=A(\vec{c}, \vec{d}) .
$$

A representação de 83 pode ser vista na Figura 23

Exemplo 3:

Voltamos agora a $\mathbb{D}$ e tomamos $V \in T_{3}^{0}(\mathbb{D})$ dado por $V: \mathbb{D} \times \mathbb{D} \times \mathbb{D} \rightarrow V_{L^{3}}$

$$
V(\cdot, \cdot, \cdot)=\left|\epsilon_{i j k} \overline{d x}^{i}[\cdot] \otimes \overline{d x}^{j}[\cdot] \otimes \overline{d x}^{k}[\cdot]\right|,
$$

onde $\epsilon_{i j k}$ é o símbolo de Levi-Civita em três dimensões [16]. Atuando com $V(\cdot, \cdot, \cdot)$ na tripla de vetores deslocamento $\vec{a}, \vec{b}, \vec{c}$ ficamos com,

$$
\begin{aligned}
V(\vec{a}, \vec{b}, \vec{c}) & =\left|\epsilon_{i j k} \overline{d x}^{i}[\vec{a}] \otimes \overline{d x}^{j}[\vec{b}] \otimes \overline{d x}^{k}[\vec{c}]\right| \\
& =\left|\epsilon_{i j k} a^{i} b^{j} c^{k}\right|=|\vec{a} \cdot(\vec{b} \times \vec{c})| .
\end{aligned}
$$

O módulo, assim como no exemplo no Exemplo 2, foi usado para garantir que o resultado seja sempre nãonegativo. A expressão (85) também é conhecida, sendo escrita em geral como o determinante,

$$
V(\vec{a}, \vec{b}, \vec{c})=\left|\begin{array}{lll}
a^{1} & a^{2} & a^{3} \\
b^{1} & b^{2} & b^{3} \\
c^{1} & c^{2} & c^{3}
\end{array}\right| .
$$

O resultado fornece o volume do paralelepípedo definido pelos três vetores $\vec{a}, \vec{b}$ e $\vec{c}$.

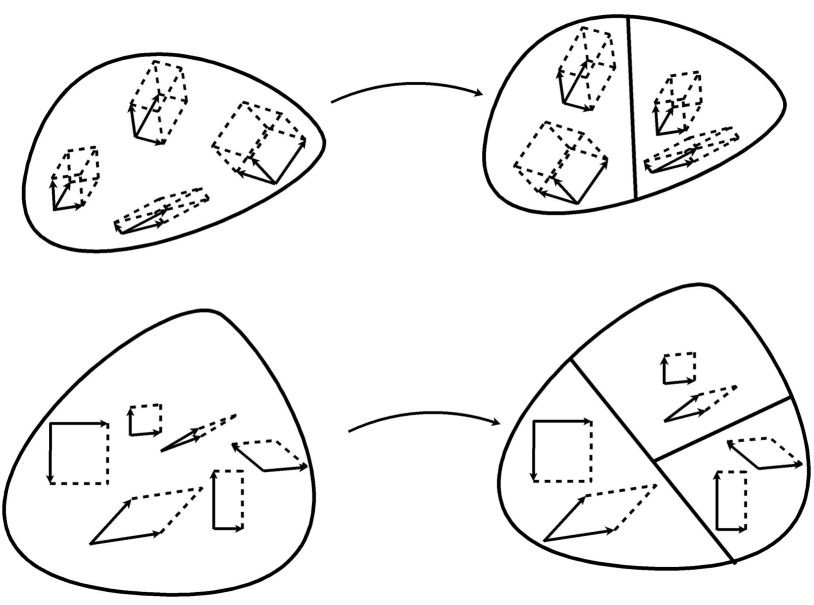

Figura 23: Domínio dos tensores "organizado" em classes. 
A grandeza física em questão corresponde ao volume. As classes de equivalência no domínio formado por trincas de vetores são definidas pela atuação de $V(\cdot, \cdot, \cdot)$, da mesma maneira que no exemplo da área, apresentado acima (veja a Figura 23):

$$
(\vec{a}, \vec{b}, \vec{c}) \sim_{V}(\vec{d}, \vec{e}, \vec{f}) \Longleftrightarrow V(\vec{a}, \vec{b}, \vec{c})=V(\vec{d}, \vec{e}, \vec{f}) .
$$

O valor da grandeza é obtido atuando $\operatorname{com} V(\cdot, \cdot, \cdot)$ na trinca correspondente.

Para concluir essa seção, vamos fazer uma lista de comentários a partir da observação dos exemplos apresentados.

1) Fixado $l \in T_{s}^{r}(\mathbb{D})$, o domínio do mapeamento corresponde ao próprio domínio da grandeza física. Por exemplo, área e volume são definidos para pares e trincas de vetores. Naturalmente tais grandezas podem ser estendidas para figuras geométricas mais gerais, sendo esta uma das motivações clássicas para o desenvolvimento do cálculo diferencial e integral.

2) As classes de equivalência em $\times_{r} \overline{\mathbb{D}} \times_{s} \mathbb{D}$ são definidas pelo valor que a grandeza $l \in T_{s}^{r}(\mathbb{D})$ assume, isto é, dados $\bar{p}_{\times r} \times \vec{a}_{\times s}$ e $\bar{q}_{\times r} \times \vec{b}_{\times s}$ em $\times_{r} \overline{\mathbb{D}} \times\left.{ }_{s} \mathbb{D}\right|^{5}$

$\bar{p}_{\times r} \times \vec{a}_{\times s} \sim_{l} \bar{q}_{\times r} \times \vec{b}_{\times s} \Leftrightarrow l\left(\bar{p}_{\times r} \times \vec{a}_{\times s}\right)=l\left(\bar{q}_{\times r} \times \vec{b}_{\times s}\right)$.

3) O espaço de valores da grandeza é obtido a partir da atuação de $l \in T_{s}^{r}(\mathbb{D})$ no domínio correspondente.

4) Os exemplos de $A(\cdot, \cdot)$ e $V(\cdot, \cdot, \cdot)$ possuem um fator em comum: os coeficientes das combinações lineares dos vetores de base foram símbolos antissimétricos. É a partir daí que nasce a teoria das formas diferenciais. Neste contexto, gostaríamos de parafrasear o Prof. João Barata, nas suas "Notas de Física Matemática" 16],

"A teoria das formas diferenciais é tão elegante que parece ter sido achada, não inventada."

Esperamos que nosso ponto de vista operacional possa reforçar tal afirmação.

5) Com o exemplo da grandeza volume, ficamos tentados a dividir nosso espaço em uma coleção de volumes de forma que cada $V_{\lambda}$ corresponda a uma trinca de vetores indexada por $\lambda \in \Lambda \subset \mathbb{R}$, sendo tal coleção fechada por uniões enumeráveis. Seria razoável também que, caso dois destes volumes não se intersectem em uma região espacial, o volume da união dos dois seja igual à soma dos volumes. Para o leitor que já estudou um pouco de matemática avançada, estamos falando de construir tanto uma $\sigma$-álgebra quanto a noção de medida para nosso espaço físico 14.

6) O exemplo do mapeamento $\Pi(\cdot, \cdot)$, junto com o Teorema 4 dota o espaço de vetores deslocamento com um produto interno, que, contrariamente ao costume nos

\footnotetext{
${ }^{5}$ Usamos a notação condensada:

$$
\bar{p}_{\times r} \times \vec{a}_{\times s}:=\left(\bar{p}_{1}, \ldots, \bar{p}_{r}, \vec{a}_{1}, \ldots, \vec{a}_{s}\right) \in \times_{r} \overline{\mathbb{D}} \times_{s} \mathbb{D} .
$$
}

livros de matemática ${ }^{6}$ foi induzido pelo módulo

$$
\Pi(\vec{a}, \vec{a})=|\vec{a}|^{2} .
$$

Ora, se temos módulos de vetores, definidos por compassos, nasce também a possibilidade de construir conjuntos abertos em $\mathbb{D}$ a partir das esferas $\mathcal{B}(O, d(A, B))$ previamente definidas. Neste caso, dotamos $\mathbb{D}$ e por sua vez $\mathcal{E}_{S R}$ com uma estrutura topológica. Planejamos discutir a formulação operacional da topologia do espaço futuramente.

7) O último comentário é técnico, mas imprescindível. Quando construímos o produto tensorial, não nos preocupamos em garantir, por exemplo, associatividade. Isto é, dados espaços vetoriais $V_{1}, V_{2}$ e $V_{3}$, não discutimos se é possível escrever

$$
\left(V_{1} \otimes V_{2}\right) \otimes V_{3} \cong V_{1} \otimes\left(V_{2} \otimes V_{3}\right),
$$

onde $\cong$ significa isomorfismo. Para garantir o fato acima, embasando a definição de tensores, façamos uma breve digressão sobre o que os matemáticos chamam de propriedade de universalidade. Primeiramente, denotaremos por $\mathbb{K}$ o corpo sobre qual cada espaço vetorial está definido. Nos contextos da física, tem-se geralmente $\mathbb{R}$ ou $\mathbb{C}$. Em seguida, denotaremos por $U$ e $V$ espaços vetoriais de dimensão finita sobre o corpo $\mathbb{K}$. O chamado espaço dual de $V$, que aqui denotaremos por $V^{*}$, é definido como sendo o conjunto formado pelos funcionais lineares de $V$ munido das operações usuais de soma e multiplicação por escalar. É um resultado conhecido que $V \cong V^{*}$ de maneira não canônica, ou seja, o isomorfismo depende da escolha da base. Em contraste, tem-se que $V$ é isomorfo ao seu bidual $V^{* *}$ de maneira canônica. Após essa identificação, costuma-se definir o produto tensorial entre os espaços $U$ e $V$ por

$$
U \otimes V:=\left\{\phi: U^{*} \times V^{*} \rightarrow \mathbb{K} \mid \phi \text { bilinear }\right\} .
$$

Chamando de Hom (homomorfismo) o conjunto de operadores lineares entre espaços vetoriais, temos o seguinte

Teorema 5 (Propriedade de Universalidade). Sejam U, $V$ e $W \mathbb{K}$-espaços vetoriais de dimensão finita. Para cada aplicação bilinear $\phi \in \operatorname{Hom}(U, V ; W)$, existe uma uma única aplicação $\psi \in H o m(U \otimes V ; W)$ tal que

$$
\phi(\vec{u}, \vec{v})=\psi(\vec{u} \otimes \vec{v}) .
$$

Portanto, tem-se o seguinte isomorfismo canônico,

$$
H o m(U, V ; W) \cong H o m(U \otimes V ; W) .
$$

O resultado acima mostra que o produto tensorial é único a menos de isomorfismo. Podemos ilustrar a propriedade

\footnotetext{
${ }^{6}$ Em geral, o módulo (norma) é menos restritivo que o produto interno. Um espaço vetorial dotado de um produto interno é naturalmente dotado de um módulo mas não o inverso 11 .
} 
de universalidade através do seguinte diagrama comutativo

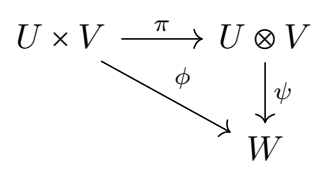

onde $\pi(\vec{u}, \vec{v})=\vec{u} \otimes \vec{v}$. No caso de $W=\mathbb{K}$ a propriedade universal nos diz que $\operatorname{Hom}(U, V ; \mathbb{K})$ é isomorfo a $(U \otimes$ $V)^{*}$. Neste momento devemos lembrar do começo da nossa discussão. O espaço vetorial $V$ pode ser identificado com o seu bidual e isso nos permite concluir o seguinte resultado:

$$
U^{*} \otimes V^{*} \cong(U \otimes V)^{*}
$$

A este ponto, esperamos que o leitor esteja convencido da importância da propriedade universal do produto tensorial. Entretanto, esta se mostra extremamente útil no que diz respeito a outras propriedades do produto tensorial, algumas das quais mencionaremos agora. Gostaríamos que algumas propriedades rotineiras continuassem sendo válidas, por exemplo,

(i) (Associatividade): $(U \otimes V) \otimes W \cong U \otimes(V \otimes W)$;

(ii) (Comutatividade): $U \otimes V \cong V \otimes U$;

(iii) (Distributividade): $U \otimes(V \oplus W) \cong U \otimes V \oplus U \otimes W$.

Em (iii), pedimos distributividade em relação à soma direta. Aqui é necessário uma grande atenção. Num produto tensorial do tipo $V \otimes V$ não podemos dizer que em geral tem-se $\vec{u} \otimes \vec{v}=\vec{v} \otimes \vec{u}$ ! De fato, por exemplo,

$$
\hat{i} \otimes \hat{j}(d x, d y)=d x(\hat{i}) d y(\hat{j})=1 \cdot 1=1 .
$$

Por outro lado,

$$
\hat{i} \otimes \hat{j}(d y, d x)=d y(\hat{i}) d x(\hat{j})=0 \cdot 0=0,
$$

que nos permite concluir que $\hat{i} \otimes \hat{j} \neq \hat{j} \otimes \hat{i}$. A propriedade (ii) nos diz apenas que a propriedade de comutatividade existe para produtos tensoriais de espaços mas não para produto de vetores! Por fim, vale a pena destacar que a propriedade de associatividade significa

$$
\begin{aligned}
(U \otimes V) \otimes W & \cong\left\{\phi: U^{*} \times V^{*} \times W^{*} \rightarrow \mathbb{K} \mid \phi \text { trilinear }\right\} \\
& \cong U \otimes(V \otimes W),
\end{aligned}
$$

e isto nos permite definir $U \otimes(V \otimes W):=U \otimes V \otimes W$. Indutivamente, concluímos que para $V_{1}, \ldots, V_{n}$ espaços vetoriais tem-se

$$
V_{1} \otimes \cdots \otimes V_{n} \stackrel{!}{=}\left\{\phi: V_{1}^{*} \times \cdots \times V_{n}^{*} \rightarrow \mathbb{K} \mid \phi \text { n-linear }\right\} .
$$

O trabalho "duro" relacionado à parte matemática termina aqui e agora podemos voltar ao nosso mundo das grandezas físicas. Neste ponto, já podemos concluir que a definição dada para $T_{s}^{r}(\mathbb{D})$ está bem construída, graças à propriedade (95). Não há ambiguidade em definirmos o espaço dos tensores do tipo $(r, s)$, como na Definição 10 . Vale a pena destacar que o uso da propriedade de univer- salidade para definir o produto tensorial é preferida pela comunidade matemática, sobretudo aqueles que trabalham com Álgebra, e é pouco comum na Física. Grande parte dos textos em física costumam definir tensores como objetos que sob mudança de base se comportam de uma determinada forma, escondendo, com isso, uma formulação matemática limpa e conectada com o mundo físico.

\section{Conclusão}

Apresentamos ao longo desse trabalho a construção operacional de grandezas físicas multidimensionais, a saber, vetores, vetores duais e tensores. O trabalho foi recheado com introduções heurísticas para cada grandeza, culminando com a sua devida formalização matemática e operacional. Devido ao caráter geométrico de tais grandezas, ressignificamos também alguns tópicos de matemática, que agora passamos a listar.

1. Toda geometria foi reformulada. Para um operacionalista, é inadmissível tratar pontos, retas e planos como conceitos primitivos. Aqui eles foram definidos.

2. Os matemáticos resumem qualquer espaço vetorial real como $\mathbb{R}^{n}$. Muitos físicos, por sua vez, misturam estruturas distintas como espaço físico $\left(\mathcal{E}_{S R}\right)$, espaço de vetores deslocamento $(\mathbb{D})$ e $\mathbb{R}^{3}$ como se fossem a mesma coisa. Aqui deixamos clara tal distinção. Além disso, os físicos têm preferência por espaços que possuam uma estrutura de um espaço afim. Em contraste com $\mathbb{R}^{n}$, a estrutura afim pode ser naturalmente usada para descrever a homogeneidade e isotropia do espaço. De um certo ponto de vista o espaço $\mathbb{R}^{n}$ possui uma estrutura "não física", ou seja, ela quebra essas simetrias no sentido que admite um sistema de coordenadas "preferencial".

3. Com o exemplo do tensor $\Pi(\cdot, \cdot)$ na Seção 6 , fica claro como é possível induzir um produto interno a partir de uma norma. Essa direção é contrária ao que ocorre usualmente na matemática. Além disso, como o compasso gera naturalmente o conceito de bolas abertas, podemos tratar a estrutura topológica do espaço sob o ponto de vista operacional, como citado.

Como mencionamos anteriormente, a escola operacional pode ser um tanto quanto controversa. Esperamos, contudo, que esse artigo colabore para a conexão de estruturas matemáticas puras para a descrição do mundo que tocamos, apresentando a temática associada a construção de grandezas físicas.

\section{Agradecimentos}

Este trabalho foi financiado pelo Programa Institucional de Bolsas de Iniciação Científica - XXXI BIC/UFJF$2018 / 2019$. 


\section{Referências}

[1] G.F. Vasconcelos Júnior, R.P.S. Costa e B.F. Rizzuti, Rev. Bras. Ensino Fís. 40, e3304 (2018).

[2] https://www.bipm.org/utils/common/documents/ jcgm/JCGM_200_2012.pdf.

[3] P.W. Bridgman, Logic of modern physics (MacMillan, New York, 1927).

[4] <https://plato.stanford.edu/archives/fall2009/ entries/operationalism/>, acessado em 10/19/2018.

[5] J.M. Jauch, Foundations of Quantum Mechanics (Addison-Wesley Publ., Reading, 1968).

[6] E.Q.F. Rezende e M.L.B. Queiroz, Geometria Euclidiana Plana e Construções Geométricas (Editora da Unicamp, Campinas, 2000).

[7] <http://www.fisica.ufjf.br/ lesche/>, acessado em $12 / 09 / 2018$.

[8] P.R. Halmos, Teoria ingênua dos conjuntos (Editora Ciência Moderna, Rio de Janeiro, 2001).

[9] B. Lesche, Teoria da Relatividade (Editora Livraria da Física, São Paulo, 2005).

[10] A.N. Rocha, B.F. Rizzuti e D.S. Mota, Revista Brasileira de Ensino de Física 35, 4304 (2013).

[11] D.P. Pombo Jr., Introdução à Análise Funcional (Editora da UFF, Niterói, 2009).

[12] K.M. Hoffman and R. Kunze, Linear Algebra (PrenticeHall, New Jersey, 1971).

[13] S. Floquet, A.A. Castro Júnior, M.A.S. Trindade e J.D.M. Vianna, Revista Brasileira de Ensino de Física 40, e3303 (2018).

[14] N.A. Lemos, Convite à Física Matemática (Editora Livraria da Física, São Paulo, 2013).

[15] http://www.cidepe.com.br/index.php/br/, acessado em 18/09/2018.

[16] http://denebola.if.usp.br/ jbarata/Notas_ de_aula/arquivos/nc-cap37.pdf, acessado em $19 / 09 / 2018$. 